\title{
Oscillations of solar and atmospheric neutrinos
}

\author{
R. Barbieri ${ }^{1}$, L.J. Hall ${ }^{2}$, D. Smith ${ }^{2}$, A. Strumia ${ }^{3}$ and N. Weiner ${ }^{2}$ \\ 1 Scuola Normale Superiore, and \\ INFN, sezione di Pisa, I-56126 Pisa, Italia \\ 2 Department of Physics and \\ Theoretical Physics Group, Lawrence Berkeley National Laboratory \\ University of California, Berkeley, California 94720 \\ 3 Dipartimento di fisica, Università di Pisa, and \\ INFN, sezione di Pisa, I-56127 Pisa, Italia
}

\begin{abstract}
Motivated by recent results from SuperKamiokande, we study both solar and atmospheric neutrino fluxes in the context of oscillations of the three known neutrinos. We aim at a global view which identifies the various possibilities, rather than attempting the most accurate determination of the parameters of each scenario. For solar neutrinos we emphasise the importance of performing a general analysis, independent of any particular solar model and we consider the possibility that any one of the techniques - chlorine, gallium or water Cerenkov - has a large unknown systematic error, so that its results should be discarded. The atmospheric neutrino anomaly is studied by paying special attention to the ratios of upward and downward going $\nu_{e}$ and $\nu_{\mu}$ fluxes. Both anomalies can be described in a minimal scheme where the respective oscillation frequencies are widely separated or in non-minimal schemes with two comparable oscillation frequencies.

We discuss explicit forms of neutrino mass matrices in which both atmospheric and solar neutrino fluxes are explained. In the minimal scheme we identify only two 'zeroth order' textures that can result from unbroken symmetries. Finally we discuss experimental strategies for the determination of the various oscillation parameters.
\end{abstract}

\footnotetext{
${ }^{\dagger}$ This work was supported in part by the U.S. Department of Energy under Contracts DE-AC03-76SF00098, in part by the National Science Foundation under grant PHY-95-14797, in part by the TMR network under the EEC contract n. ERBFMRXCT960090.
} 


\section{Introduction}

The solar and atmospheric neutrino flux anomalies have both been considerably strengthened by recent observations from Super-Kamiokande. The solar neutrino flux is measured to be [1] $0.37 \pm 0.03$ of that expected from the 'BP95' standard solar model [2], without including any theoretical error. This is the fifth solar neutrino experiment to report results in strong disagreement with the predictions of solar models. Furthermore, using a solar model independent analysis, the measured solar fluxes are found to be in conflict with each other. For events at SuperKamiokande with visible energies of order a $\mathrm{GeV}$, the ratio of 1 ring $\mu$-like to $e$-like events is $0.66 \pm 0.10$ that expected from calculations of the flux of neutrinos produced in the atmosphere in cosmic ray showers [3]. Furthermore, the distribution in zenith angle of these 1 ring events provides striking evidence for a depletion of $\nu_{\mu}$ which depends on the distance travelled by the neutrinos before reaching the Super-Kamiokande detector. In particular, the observed up/down ratio of the multi-GeV, $\mu$-like events is $0.52 \pm 0.07$. This significantly strengthens the evidence that $\nu_{\mu}$ oscillate as they traverse the earth.

In this paper, we interpret the solar and atmospheric neutrino flux anomalies in terms of oscillations of the three known neutrinos $\nu_{e, \mu, \tau}$. The lightness of these three neutrinos, relative to the charged fermions, can be simply understood as resulting from large $\mathrm{SU}(2)_{L} \otimes \mathrm{U}(1)_{Y}$ invariant masses for the right-handed neutrinos, via the see-saw mechanism. We do not consider the possibility of a fourth light neutrino, as it would have to be singlet under $\mathrm{SU}(2)_{L} \otimes \mathrm{U}(1)_{Y}$, and would either require a new mass scale far below the weak scale, running counter to the idea of the see-saw mechanism, or a more complicated see-saw.

Theoretical ideas about generation mixing are guided by the quark sector, where the mixing angles are all small, indicating a hierarchical breaking of horizontal symmetries in nature. A similar hierarchy of horizontal symmetry breaking in the lepton sector is also likely to yield small angles, suggesting small probabilities for a neutrino to oscillate from one flavour to another. However, the solar and atmospheric neutrino flux measurements both require neutrino survival probabilities, $P_{e e}$ and $P_{\mu \mu}$, far from unity. Over a decade ago 何, it was realised that large angles were not necessary to account for the large suppression of solar neutrino fluxes while $\nu_{e}$ have charged current interactions in the solar medium, $\nu_{\mu, \tau}$ do not, allowing a level crossing phenomena where a $\nu_{e}$ state produced in the solar interior evolves to a $\nu_{\mu, \tau}$ state as it traverses the sun. This simple picture can reconcile the three types of solar neutrino flux measurements with the standard solar model, for a mixing angle as small as 0.03 - a significant achievement. Could such resonant oscillations occur for atmospheric neutrinos in the earth, again allowing a small vacuum mixing angle? In this case, since the earth does not have a continuously varying density, the matter mixing angle in the earth is much larger than the vacuum mixing angle only in a small range of energies. Hence, an oscillation interpretation of the atmospheric neutrino fluxes requires a large mixing angle, and calls into question the frequently stated theoretical prejudice in favour of small mixing angles.

In this paper, we attempt to understand both solar and atmospheric neutrino fluxes using 3-generation neutrino oscillations, aiming at a global view which identifies the various possibilities, rather than attempting the most accurate determination of the parameters of each scenario. When data from chlorine, gallium and water Cerenkov detectors are fitted to a standard solar model, standard analyses find very small regions of neutrino mass and mixing parameters. For 2-generation mixing, these are known as the "small angle MSW", "large angle MSW" and "just so" regions. This analysis has been extended to the case of three generations [5], with a single matter resonance in the sun, as suggested by the atmospheric neutrino data. The large and small angle MSW areas are found to merge into a single MSW volume of parameter space. In subsection 2.1, we study how this volume is enlarged when a solar model independent analysis of the solar fluxes replaces the use of a single solar model. In subsection 2.2 we extend our analysis to see what areas of neutrino parameter space become allowed if one of the three observational techniques to measure the solar fluxes is seriously in error.

We combine these regions of parameters with those yielding the atmospheric fluxes, and find there is still considerable allowed ranges of masses and mixing angles. This is done in section 3 , assuming that the smallest of the two neutrino squared mass differences is too small to affect the oscillations of atmospheric neutrinos (minimal scheme). In section 1 , on the contrary, we allow for the possibility that the two independent neutrino squared mass differences are both large enough to affect atmospheric neutrino oscillations (non minimal schemes). For solar neutrinos, this requires that there is a serious flaw either in at least one measurement technique or in solar model analyses.

The forms of neutrino mass matrices that can lead to a large $\nu_{\mu} \rightleftharpoons \nu_{\tau}$ mixing for atmospheric neutrinos are discussed in section 5. In section 6 only two 'zeroth order' textures for neutrinos masses are identified that can account for the atmospheric and solar neutrino data in the minimal scheme and can result from unbroken symmetries.

Our conclusions are drawn in section 0 . Based on a simple set of alternative hypotheses, we discuss how future measurements could eventually determine the two neutrino mass differences and the three mixing angles. 


\section{Solar neutrinos: model-independent analysis}

In the flavour eigenstate basis, in which the charged lepton mass matrix is diagonal, the neutrino mass matrix is in general non-diagonal. It may be diagonalized by a unitary transformation:

$$
\nu_{f}=V_{f i}^{*} \nu_{i}
$$

where $\nu_{f}$ and $\nu_{i}$ are flavour and mass eigenstate fields, respectively. The leptonic analogue of the CabibboKobayashi-Maskawa mixing matrix is $V^{T}$, since the $W$ boson couples to the charged current $\bar{\nu}_{i_{L}} V_{i f}^{T} \gamma^{\mu} e_{f_{L}}$. In addition to the three Euler angles, $V$ contains physical phases: one if the light neutrinos are Dirac, and three if they are Majorana. These flavour and mass eigenstate fields destroy basis states which are related by

$$
\left|\nu_{f}\right\rangle=V_{f i}\left|\nu_{i}\right\rangle
$$

If some process creates a flavour eigenstate, $\left|\nu_{f}\right\rangle$, at time $t=0$, then at a later time $t$ it will have evolved to the state $\left|\nu_{f}, t\right\rangle=\psi_{f^{\prime}}(t)\left|\nu_{f^{\prime}}\right\rangle$ via the matrix Schroedinger equation

$$
i \frac{d \psi}{d t}=\left(V \frac{m_{\nu}^{2}}{2 E} V^{\dagger}+A_{\mathrm{CC}}+\mathcal{E}\right) \psi
$$

where $E$ is the energy of the relativistic neutrino, $m_{\nu}$ is the diagonal neutrino mass matrix with entries $m_{i}, \mathcal{E}$ is an irrelevant term proportional to the unit matrix, and $A_{\mathrm{CC}}$ represents matter effects. For neutrinos propagating in matter with electron number density $N_{e}, A_{\mathrm{CC}}$ is a matrix with a single non-zero entry, $A_{\mathrm{CC}}^{11}=\sqrt{2} G_{F} N_{e}$.

The mixing matrix $V$ can be written quite generally as

$$
V=R_{23}\left(\theta_{23}\right)\left(\begin{array}{ccc}
1 & 0 & 0 \\
0 & e^{i \phi} & 0 \\
0 & 0 & 1
\end{array}\right) R_{13}\left(\theta_{13}\right) R_{12}\left(\theta_{12}\right)\left(\begin{array}{ccc}
1 & 0 & 0 \\
0 & e^{i \alpha} & 0 \\
0 & 0 & e^{i \beta}
\end{array}\right)
$$

where $R_{i j}\left(\theta_{i j}\right)$ represents a rotation by $\theta_{i j}$ in the $i j$ plane. We have chosen a sequence of rotations which frequently arises in the diagonalization of simple hierarchical forms for the neutrino mass matrix, as illustrated in section 6. From equation (2.3) we see that the phases $\alpha$ and $\beta$ never appear in oscillation phenomena, and hence can be dropped, giving

$$
V=\left(\begin{array}{ccc}
c_{12} c_{13} & c_{13} s_{12} & s_{13} \\
-c_{23} s_{12} e^{i \phi}-c_{12} s_{13} s_{23} & c_{12} c_{23} e^{i \phi}-s_{12} s_{13} s_{23} & c_{13} s_{23} \\
s_{23} s_{12} e^{i \phi}-c_{12} c_{23} s_{13} & -c_{12} s_{23} e^{i \phi}-c_{23} s_{12} s_{13} & c_{13} c_{23}
\end{array}\right)
$$

Each $R_{i j}$ must diagonalize a symmetric $2 \times 2$ sub-matrix determining $\tan 2 \theta_{i j}$, hence, without loss of generality, we may choose $0 \leq \theta_{i j} \leq \pi / 2$, while $0 \leq \phi<2 \pi$. A more convenient choice is to keep $\theta_{12,13}$ in the first quadrant, while $0 \leq \theta_{23}, \phi \leq \pi$. We choose to order the neutrino mass eigenstates so that $\Delta m_{23}^{2}>\Delta m_{12}^{2}>0$, where $\Delta m_{i j}^{2} \equiv m_{i}^{2}-m_{j}^{2}$. Notice that with this parametrization $V_{e 3} \ll 1$ means $\theta_{13}$ close to 0 or to $90^{\circ}$.

To study solar neutrinos, we are interested only in the electron neutrino survival probability, $P_{e e}$, and hence in the evolution of $\psi_{e}$. This evolution does not depend on $\theta_{23}$ or on $\phi-$ on substituting (2.4) in (2.3), $R_{23}$ and $\phi$ can be absorbed into redefined states $\mu^{\prime}$ and $\tau^{\prime}$. Hence, we have shown quite generally that $P_{e e}$ depends only on four neutrino parameters: $\Delta m_{12}^{2}, \Delta m_{23}^{2}, \theta_{12}$ and $\theta_{13}$.

For an oscillation explanation of the atmospheric neutrino fluxes, $\Delta m_{23}^{2}$ is sufficiently large that it does not cause a resonance transition in the sun. In the Landau-Zehner approximation, the evolution equation (2.3) can be solved to give [6]

$$
P_{e e}=\left(\left|V_{e 1}\right|^{2},\left|V_{e 2}\right|^{2},\left|V_{e 3}\right|^{2}\right)\left(\begin{array}{ccc}
1-P & P & 0 \\
P & 1-P & 0 \\
0 & 0 & 1
\end{array}\right)\left(\begin{array}{c}
\left|V_{e 1}^{\mathrm{m}}\right|^{2} \\
\left|V_{e 2}^{\mathrm{m}}\right|^{2} \\
\left|V_{e 3}^{\mathrm{m}}\right|^{2}
\end{array}\right)
$$

where $V_{e i}^{\mathrm{m}}$ are the mixing matrix elements in matter, and $P$ is the transition probability between the states at resonance:

$$
P=e^{-E_{\mathrm{NA}} / E} \theta\left(E-E_{\mathrm{A}}\right), \quad E_{\mathrm{NA}}=\frac{\pi \Delta m_{12}^{2} \sin ^{2}\left(2 \theta_{12}\right)}{4\left|\frac{1}{N_{e}} \frac{d N_{e}}{d x}\right|_{1} \cos \left(2 \theta_{12}\right)}, \quad E_{\mathrm{A}}=\frac{\Delta m_{12}^{2} \cos 2 \theta_{12}}{2 \sqrt{2} G_{\mathrm{F}}\left|N_{e}\right|_{0} \cos ^{2} \theta_{13}}
$$

Here $E$ is the neutrino energy, $\theta$ is the step function, the 1 subscript indicates that $N_{e}$ and its gradient $d N_{e} / d x$ are evaluated at the resonance point, while the 0 subscript indicates the production point. The large mass splitting $\Delta m_{23}^{2}$ enters $P_{e e}$ only via the matter mixing angles, and decouples from these expressions in the limit 

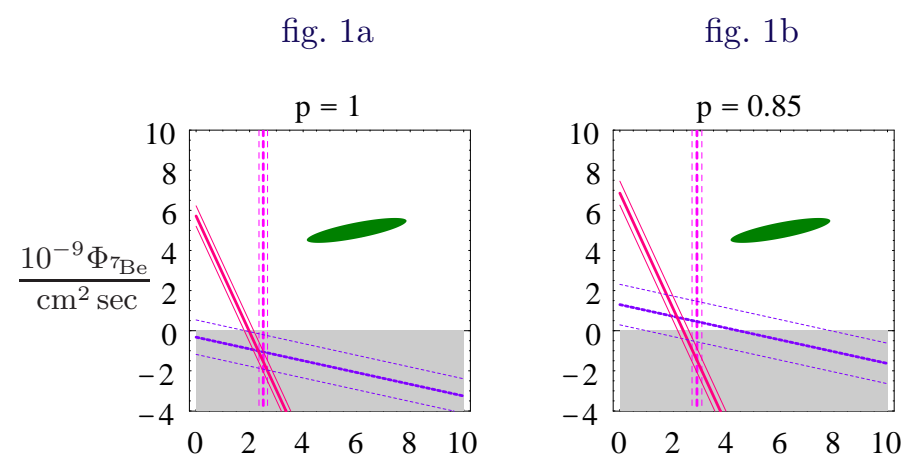

fig. 11c

fig. $\mathbb{1} \mathrm{d}$

Flux of boron neutrinos, $\Phi_{8_{\mathrm{B}}}$, in $10^{6} / \mathrm{cm}^{2} \mathrm{sec}$

Figure 1: Values of $\left(\Phi_{8_{\mathrm{B}}}, \Phi_{7_{\mathrm{Be}}}\right)$ measured by the Chlorine experiment (continuous lines), the Gallium experiment (dashed lines) and by the SuperKamiokande experiment (long dashed lines) assuming various neutrino oscillation

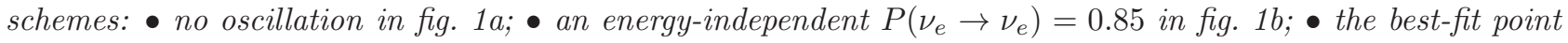
of the small-angle MSW oscillation in fig. 目c; • the best-fit point of the large-angle MSW oscillation in fig. Id.

that it is much larger than $A_{11} E$, and also in the limit that $\theta_{13}$ vanishes. For most of this section we make $\Delta m_{23}^{2}$ sufficiently large that it decouples, and we comment at the end on the effect on the allowed regions of parameter space for non-zero $\theta_{13}$ and small $\Delta m_{23}^{2}$, where $\Delta m_{23}^{2}$ effects may not decouple.

The signals $S_{i}$ at the three types of solar neutrino experiments are

$$
S_{i}=\int d E \Phi(E)\left[\sigma_{i}^{e}(E) P_{e e}(E)+\sigma_{i}^{\not}(E)\left(1-P_{e e}(E)\right)\right], \quad i=\{\mathrm{SK}, \mathrm{Ga}, \mathrm{Cl}\}
$$

where $\Phi(E)$ is the total flux of solar neutrinos with energy $E$, and $\sigma_{i}^{e, \not}(E)$ are the interaction cross sections at experiment $i$ for electron-type and non-electron-type neutrinos, respectively (only the water Cerenkov detectors are sensitive to neutral currents, so $\left.\sigma_{\mathrm{Ga}}^{\not}(E)=\sigma_{\mathrm{Cl}}^{\not}(E)=0\right)$. We will use the theoretical predictions of the various cross sections found in 7,8 . The flux $\Phi(E)$ is broken into components in the standard way by specifying the production reaction, giving [7]

$$
\Phi(E)=\sum_{\alpha} \Phi_{\alpha} f_{\alpha}(E), \quad \text { with } \quad \int_{0}^{\infty} f_{\alpha}(E) d E=1
$$

and $\alpha=\mathrm{pp}, \mathrm{pep},{ }^{7} \mathrm{Be},{ }^{13} \mathrm{~N},{ }^{15} \mathrm{O},{ }^{17} \mathrm{~F},{ }^{8} \mathrm{~B}$, hep. At this point we follow the (nearly) model-independent treatment of the fluxes described in [9] by making the following assumptions:

1. The energy dependence $f_{\alpha}(E)$ of the single components of the neutrino fluxes predicted by solar models ([7, 2] for instance) are correct. In fact the $f_{\alpha}(E)$ do not depend on the structure of the sun, and are the same in any solar model that does not introduce non-standard electroweak effects [0].

2. The overall $\Phi_{\alpha}$ can differ from their solar models predictions. However there are strong physical reasons to believe that the ratios $\Phi_{13 \mathrm{~N}} / \Phi_{15 \mathrm{O}}$ and $\Phi_{\mathrm{pep}} / \Phi_{\mathrm{pp}}$ can be set to their solar SM values [2]. Furthermore we neglect entirely hep and ${ }^{17} \mathrm{~F}$ neutrinos, which we expect to be extremely rare.

3. The present total luminosity of the sun, $K_{\odot}$, determines its present total neutrino luminosity as

$$
K_{\odot}=\sum_{\alpha}\left(\frac{Q}{2}-\left\langle E_{\nu_{\alpha}}\right\rangle\right) \Phi_{\alpha} \approx \frac{Q}{2} \sum_{\alpha} \Phi_{\alpha}
$$

where $Q=26.73 \mathrm{MeV}$ is the energy released in the reaction $4 \mathrm{p}+2 e \rightarrow{ }^{4} \mathrm{He}+2 \nu_{e}$, and $K_{\odot}=8.53 \cdot 10^{11}$ $\mathrm{MeV} \mathrm{cm} \mathrm{cm}^{-2} \mathrm{~s}^{-1}$ is the solar radiative flux at the earth. Using 2.10) amounts to assuming that the solar energy comes from nuclear reactions that reach completion, and that the sun is essentially static over the $10^{4}$ years employed by photons to random-walk out of the solar interior. 


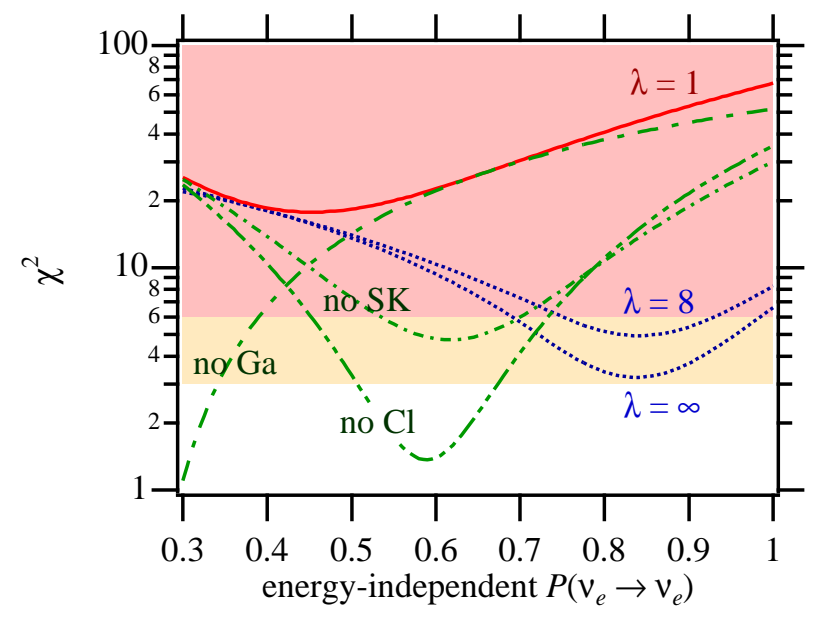

Figure 2: Values of the $\chi$-square as function of an energy independent $P\left(\nu_{e} \rightarrow \nu_{e}\right)$. The parameter $\lambda$ is defined in eq. (2.14). Also shown is the $\chi^{2}$ with one experiment discarded and $\lambda=1$.

After the first assumption we have one free parameter $\Phi_{\alpha}$ for each $\alpha$; the second then reduces the number of free parameters to four, which we can take to be

$$
\Phi_{\mathrm{p}} \equiv \Phi_{\mathrm{pp}}+\Phi_{\mathrm{pep}}, \quad \Phi_{\mathrm{CNO}} \equiv \Phi_{{ }_{13 \mathrm{~N}}}+\Phi_{15 \mathrm{O}}, \quad \Phi_{\tau_{\mathrm{Be}}} \quad \text { and } \quad \Phi_{8_{\mathrm{B}}}
$$

The luminosity constraint allows us to eliminate $\Phi_{\mathrm{p}}$, giving

$$
S_{i}=S_{i}\left(\Delta m_{12}^{2}, \theta_{12}, \theta_{13} ; \Phi_{8_{\mathrm{B}}}, \Phi_{\tau_{\mathrm{Be}}}, \frac{\Phi_{\mathrm{CNO}}}{\Phi_{{ }_{\mathrm{Be}}}}\right) .
$$

Since solar models give a stable prediction for $\Phi_{\mathrm{CNO}} / \Phi_{8_{\mathrm{B}}}=0.22$ [9], we have singled out this ratio and we will use its SSM value in our analysis. Variations of even an order of magnitude in the ratio affect negligibly our final results, since the two neutrino components have similar cross sections in existing detectors.

\subsection{Model-independent solar analysis - all experiments}

The signals now depend only on $\Phi_{8_{\mathrm{B}}}$ and $\Phi_{\mathrm{TB}_{\mathrm{B}}}$, so that, for any given oscillation pattern $P_{e e}(E)$ it is possible to plot the three experimental results: 11, 10, 11, 12

$$
\begin{aligned}
S_{\mathrm{Cl}}^{\exp } & =(2.54 \pm 0.20) 10^{-36} \mathrm{~s}^{-1} \\
S_{\mathrm{Ga}}^{\exp } & =(75 \pm 7) 10^{-36} \mathrm{~s}^{-1} \\
S_{\mathrm{SK}}^{\exp } & =(2.51 \pm 0.16) \cdot 10^{6} \mathrm{~cm}^{-2} \mathrm{~s}^{-1}
\end{aligned}
$$

as three bands in the $\left(\Phi_{8_{\mathrm{B}}}, \Phi_{7_{\mathrm{Be}}}\right)$ plane. The three bands will in general not meet, giving interesting solar model independent restrictions on the oscillations parameters.

We begin the analysis by studying the case of no neutrino oscillations $\left(P_{e e}=1\right)$. In this particular case the solar model independent analysis does not give a strong result. Surprisingly the three bands perfectly meet [9, 13) as shown in fig. 11a, but mainly in the unphysical $\Phi_{7_{\mathrm{Be}}}<0$ region, with a small area in the physical region lying within $2 \sigma$ of each central value. Since the physical crossing region has a negligible ${ }^{7} \mathrm{Be}$ flux, the value of $\Phi_{\mathrm{CNO}} / \Phi_{{ }^{7} \mathrm{Be}}$ becomes completely irrelevant.

To discuss this case in a quantitative way and to deal with more general cases it is useful to introduce the $\chi$-square function

$$
\chi_{\lambda}^{2}\left(P_{e e}\left(\Delta m_{12}^{2}, \theta_{12}, \theta_{13}\right), \Phi_{8_{\mathrm{B}}}, \Phi_{\tau_{\mathrm{Be}}}\right) \equiv \sum_{i}\left(\frac{S_{i}-S_{i}^{\exp }}{\Delta S_{i}^{\exp }}\right)^{2}+\sum_{j k} \frac{\left(\Phi_{j}-\Phi_{j}^{\mathrm{SSM}}\right)\left(\Phi_{k}-\Phi_{k}^{\mathrm{SSM}}\right)}{\lambda^{2} \Delta \Phi_{j k}^{2 \mathrm{SSM}}}
$$

where $\Delta S_{i}^{\exp }$ is the $1 \sigma$ uncertainty for experiment $i$, given in (2.13), $\Phi^{\mathrm{SSM}}$ is the flux prediction of the solar model [2] and $\Delta \Phi^{\mathrm{SSM}}$ is the corresponding error matrix, taken with some generosity. The $1 \sigma$ ranges of $\Phi_{8_{\mathrm{B}}}$ and

\footnotetext{
${ }^{1}$ The SuperKamiokande experimentalists give directly the value of the flux they measure. The other experiments involve more uncertain neutrino cross sections and prefer to give the frequency of events measured per target atom in their detector. For simplicity we have omitted this detail in the text, leaving a trivial inconsistency between eq. (2.13d) and (2.8).
} 
$\theta_{13}=0$
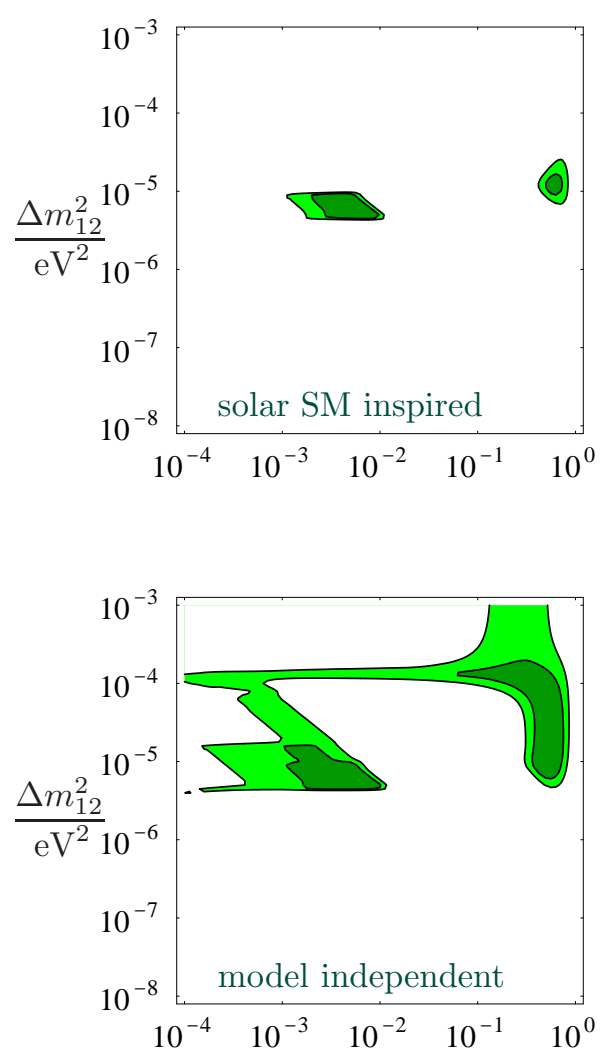

$\sin ^{2} 2 \theta_{12}$ $\theta_{13}=15^{\circ}$
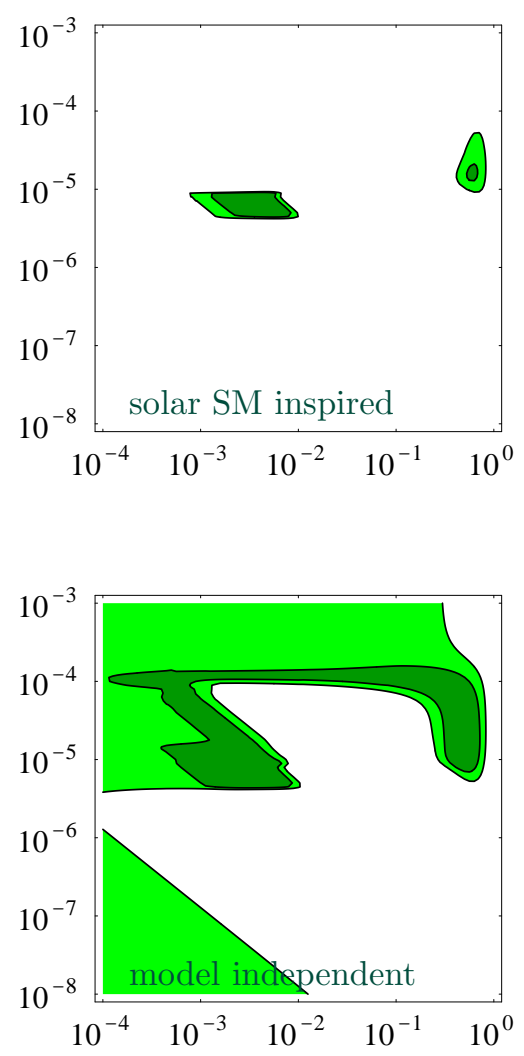

$\sin ^{2} 2 \theta_{12}$ $\theta_{13}=30^{\circ}$

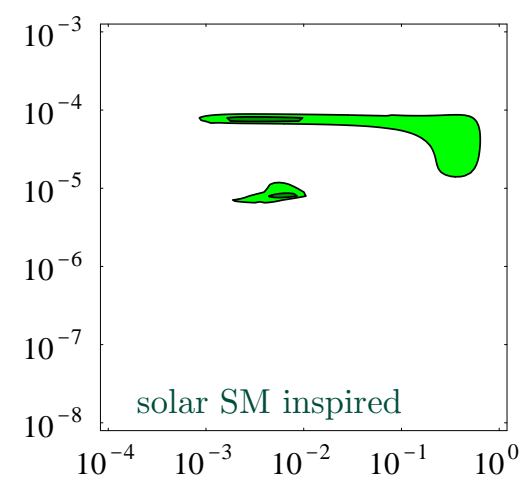

Figure 3: Allowed regions in the plane $\left(\sin ^{2} 2 \theta_{12}, \Delta m_{12}^{2}\right)$ for $\theta_{13}=0,15^{\circ}$ and $30^{\circ}$. The upper plots assume that the BP solar model is correct. The lower plots are the result of the solar model independent analysis described in the text.

$\Phi_{{ }^{\mathrm{BB}}}$ are represented by the ellipse in fig. 1. We perform our analysis with two choices for $\Delta \Phi=\lambda \cdot \Delta \Phi^{\mathrm{SSM}}$. We call the first choice, $\Delta \Phi=\Delta \Phi^{\mathrm{SSM}}$, "solar SM inspired"). The second choice, $\Delta \Phi=8 \cdot \Delta \Phi^{\mathrm{SSM}}$ ("model independent") has the same shape as the first, but is eight times as large. The part of the analysis done using this $\Delta \Phi$ is virtually free of solar physics input. The choice $\lambda=8$ (rather than $\lambda=\infty$ ) avoids unnatural values of $\Phi_{\tau_{\mathrm{Be}}}$. This choice essentially ignores solar physics considerations, but the virtue of having a number of independent experimental results is precisely that we need no longer rely heavily on solar modelling to gain insight into the underlying particle physics.

Minimizing the $\chi^{2}$ in the positive flux region we obtain $\min \chi_{8}^{2}\left(P_{e e}=1\right)=8.25$. The usual criterion for goodness of fit says that a $\chi^{2}$ with one degree of freedom larger than 8.25 is obtained with a very small probability, $\wp \approx 0.4 \%$ (a careful Monte Carlo treatment of the $\Phi_{\tau_{\mathrm{Be}}}>0$ constraint gives similar results 13 ). We however remark that, if the sun really emits the best-fit fluxes, $\Phi_{7_{\mathrm{Be}}}=0$ and $\Phi_{8_{\mathrm{B}}}=2.510^{6} / \mathrm{cm}^{2} \mathrm{~s}$, there is a $10 \%$ probability that statistical fluctuations produce the present experimental data.

We can just as easily investigate the slightly more general case of an energy independent $P_{e e}$. The dependence on the neutrino parameters $\Delta m_{12}^{2}, \theta_{12}$, and $\theta_{13}$ arises through $P_{e e}$; if the survival probability is a constant, then we can minimize $\chi_{\lambda}^{2}$ in the positive-flux region for any value of $P_{e e}$ to obtain $\min \chi_{\lambda}^{2}\left(P_{e e}\right)$, which is plotted in fig. 2 for $\lambda=1$ (SSM analysis), $\lambda=8$ (SSM independent analysis) and $\lambda=\infty$ (completely model independent analysis). For $P_{e e} \sim 0.85, \min \chi_{8}^{2}$ drops to 5 , but the fluxes required to get relatively small $\chi^{2}$ values are disfavoured by solar physics considerations $-\Phi_{\mathrm{CNO}}$ and $\Phi_{{ }_{\mathrm{BBe}}}$ must be nearly made to vanish, as shown in fig $\left[\right.$ [1 b. When $P_{e e} \lesssim 1 / 2$ the (accidental?) threefold crossing no longer occurs, so that this case can be firmly excluded in a solar-model independent way [9, 14] (see fig. 2). However, as we shall see in subsection 2.2, once we allow for the possibility that one type of experiment's results should be discarded, it is possible to obtain good fits of the data for constant $P_{e e} \sim 1 / 2$ without having to resort to unnatural flux values. 


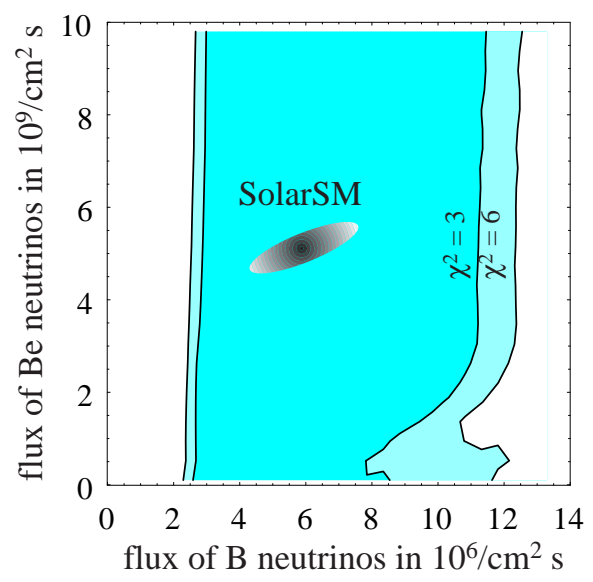

Figure 4: Isoplot of $\chi_{8}^{2}$, minimized in the mixing parameters.

Of course, we are interested in any points in parameter space that fit the data well, regardless of whether they lead to constant $P_{e e}$. For any values of $\Delta m_{12}^{2}, \theta_{12}$, and $\theta_{13}$ we can make plots similar to fig. 11a. Fig.s $1 \mathrm{c}$ and 1 d show two examples that illustrate the familiar 2-generation small and large angle MSW solutions, which evidently fit the data well if standard solar model fluxes are used.

In fig. 3 we show how the allowed regions in neutrino parameter space change if we let the fluxes vary over an expanded range of values. For each point in $\left(\Delta m_{12}^{2}, \theta_{12}, \theta_{13}\right)$ space, we minimize $\chi_{1}^{2}$ and $\chi_{8}^{2}$ by varying the fluxes within the physical region, and then we plot contours of $\min \chi_{\lambda}^{2}$ in the $\left(\sin ^{2}\left(2 \theta_{12}\right), \Delta m_{12}^{2}\right)$ plane for various values of $\theta_{13}$. The results for the "SSM inspired" and "model independent" analyses are shown in fig.s 3 (upper row and lower row, respectively). The contours are for $\chi^{2}=3$ and $\chi^{2}=6$.

For small $\theta_{13}$ the "SSM inspired" results show the standard small and large angle MSW regions. For larger values of $\theta_{13}$, the two MSW regions join, and, as $\theta_{13}$ approaches $\pi / 4$, the solutions with large $\theta_{12}$ disappear. For $\theta_{13}=\pi / 4$ the region with $\min \chi_{1}^{2}<3$ is in fact absent entirely.

The "model independent" results similarly exhibit a very strong $\theta_{13}$ dependence. We see that the "model independent" analysis continues to give strong restrictions of the oscillation parameters - in particular the $\Delta m_{12}^{2}$ values with $\min \chi_{8}^{2}<3$ are always in the range $\sim 10^{-(4 \div 5)} \mathrm{eV}^{2}$. This will not remain true when we consider the consequences of ignoring one experiment's data in subsection 2.2 .

If $\Phi_{\mathrm{CNO}} / \Phi_{8_{\mathrm{B}}}$ is ten times larger than in SSM there are new allowed regions. However these possible new regions, with $\Delta m^{2}=10^{-(5 \div 6)} \mathrm{eV}^{2}$ and $\sin ^{2} 2 \theta_{12} \gtrsim 10^{-2}$, are excluded in a model-independent way by the non observation of a day/night asymmetry at SuperKamiokande [1. 15. The recent data 1] on this asymmetry in fact disfavour as well the large angle MSW solution of the SSM-inspired analysis. Moreover, we have not included in our $\chi^{2}$ analysis the SuperKamiokande measurement of the distortion of the ${ }^{8} \mathrm{~B}$ spectrum [1, 15], because the present positive $1 \sigma$ signal could be produced by a $\Phi_{\mathrm{hep}} / \Phi_{{ }_{8 \mathrm{~B}}}$ ratio 15 times larger than the prediction of BP95 [2]. Without a very large hep flux, the present measurement excludes an otherwise allowed region with $\Delta m^{2} \approx 10^{-4} \mathrm{eV}^{2}$ and $\sin ^{2} 2 \theta_{12}$ in the range $10^{-4} \div 10^{-1}$ [13, 16 .

Our model independent analysis allows us to investigate how well present experiments are able to measure the SSM-independent neutrino fluxes $\Phi_{8_{\mathrm{B}}}$ and $\Phi_{{ }_{\mathrm{B} e}}$. This question is answered in fig. 1 , where we plot the values of the fluxes that can give a good $\left(\chi_{8}^{2}<6\right)$ or very good $\left(\chi_{8}^{2}<3\right)$ fit for some value of the oscillation parameters $\Delta m_{12}^{2}, \theta_{12}$ and $\theta_{13}$. We see that the value of $\Phi_{8_{\mathrm{B}}}$ is currently determined with an error larger than the solar model expectation. It will be directly measured in the new on-going SNO experiment. On the contrary the value of $\Phi_{{ }^{\mathrm{BBe}}}$ is at present totally unknown: in fact in the small angle MSW solution the monochromatic ${ }^{7} \mathrm{Be}$ flux can be completely converted into $\notin$ neutrinos, that are not detected by existing experiments. Borexino will be able to detect neutral currents effects in this range of energies and probably allow a direct determination of $\Phi_{\tau_{\mathrm{B} e}}$ [13].

As discussed above we perform our analysis under the assumption that $\Delta m_{23}^{2}$ is large enough that its effects decouple. For any given $\Delta m_{23}^{2}$ it is straightforward to reproduce fig. 3 by using the exact expressions for $\theta_{12}^{\mathrm{m}}$ and $\theta_{13}^{\mathrm{m}}$ in equation (2.6). In this way we find that for small $\theta_{13}\left(\lesssim 15^{\circ}\right)$, our results are insensitive to $\Delta m_{23}^{2}$ down to $\Delta m_{23}^{2}=5 \cdot 10^{-4} \mathrm{eV}^{2}$. For large $\theta_{13}, \Delta m_{23}^{2}$ effects start to become noticeable when $\Delta m_{23}^{2}$ drops below $2 \times 10^{-3} \mathrm{eV}^{2}$; for example, for $\theta_{13}=40^{\circ}$ and $\Delta m_{23}^{2}=5 \cdot 10^{-4} \mathrm{eV}^{2}$, the allowed region in the SM inspired analysis is significantly smaller than in the decoupled limit, with the $\chi_{\min }^{2}<6$ region never reaching $\sin ^{2}\left(2 \theta_{12}\right)>0.1$. In spite of these changes for small $\Delta m_{23}^{2}$, the essential features of fig. 3 in any case remain unchanged. 
$\theta_{13}=0$
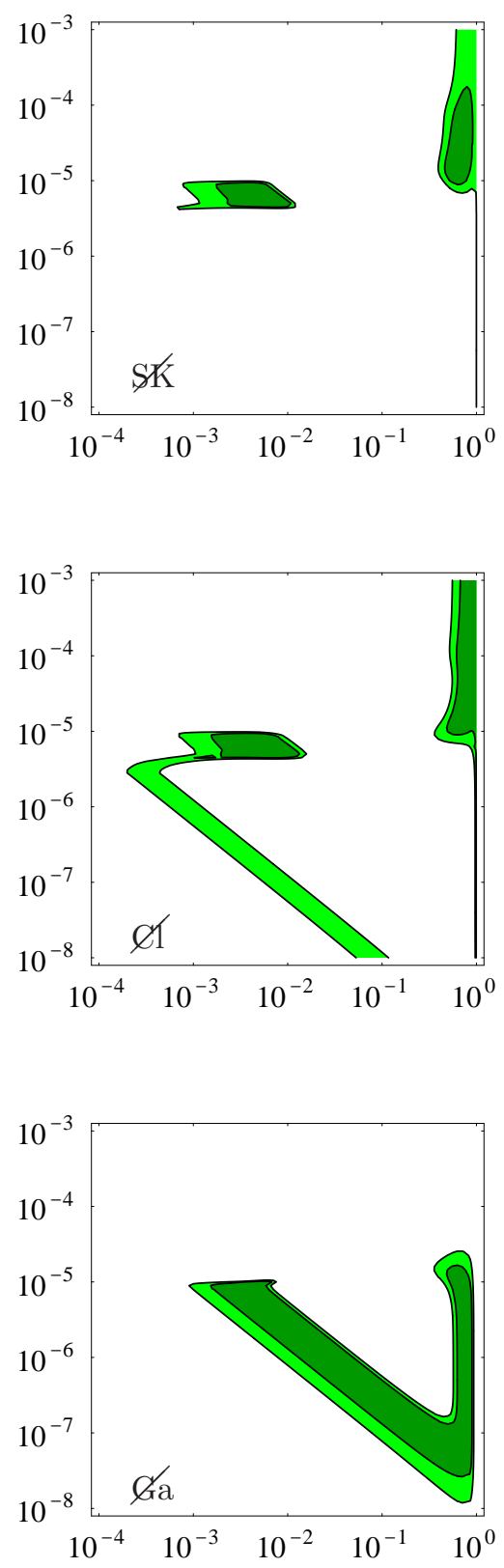

$\theta_{13}=15^{\circ}$
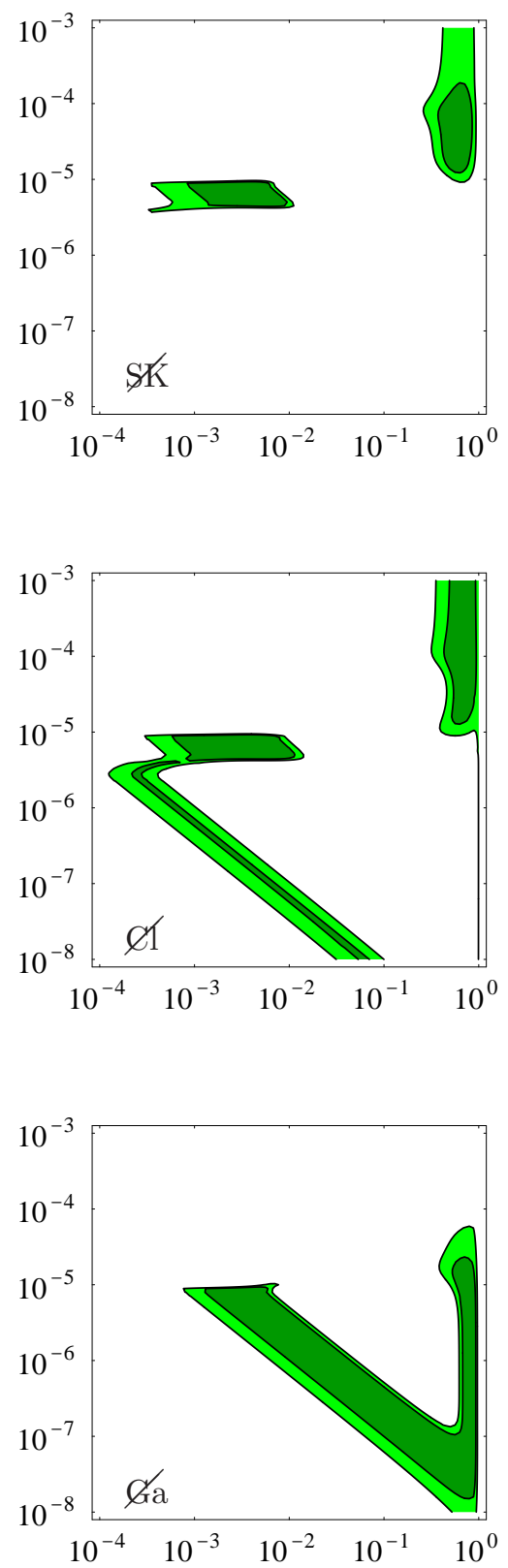

$\theta_{13}=30^{\circ}$
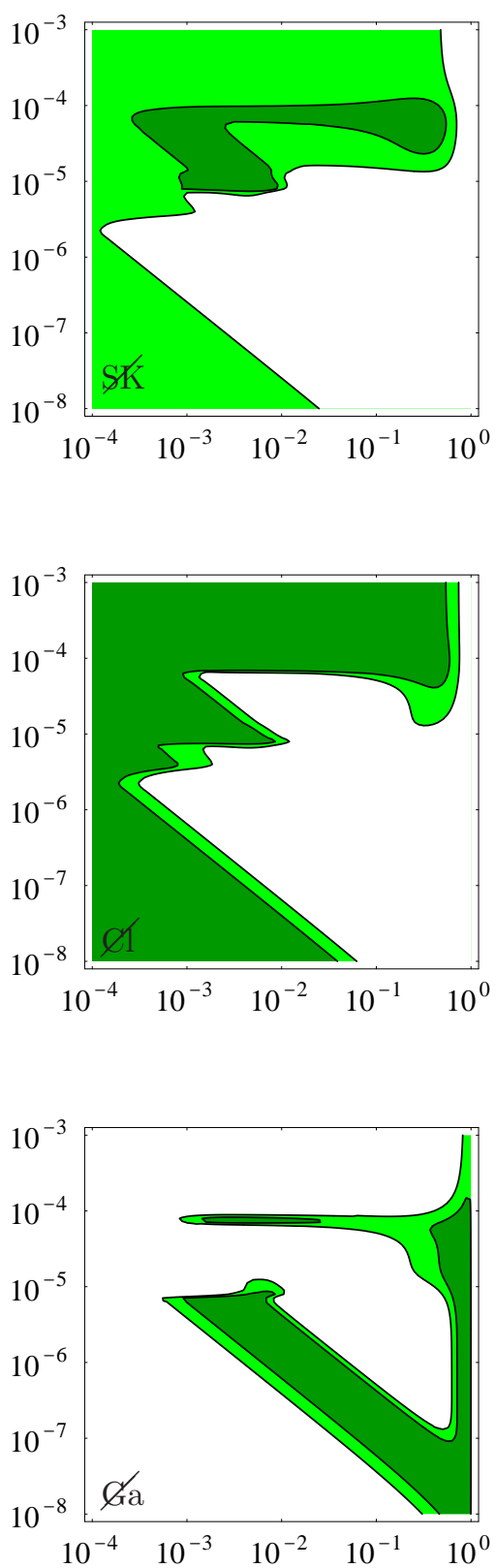

Figure 5: fits of the solar data in the plane $\left(\sin ^{2} 2 \theta_{12}, \Delta m_{12}^{2} / \mathrm{eV}^{2}\right)$ for $\theta_{13}=0,15^{\circ}$ and $30^{\circ}$ assuming that one of the three solar neutrino experiments has a large unknown systematic error (SuperKamiokande in the first row, Chlorine in the second and Gallium in the third) and is therefore discarded from the analysis. The contours are for $\chi^{2}=3$ and $\chi^{2}=6$. 


\subsection{Model independent solar analysis - one experiment ignored}

In subsection 2.1, the present level of experimental evidence allowed us to omit one restriction (the solar model) and still yield interesting results. Likewise, we can choose to omit one experiment from the analysis while keeping some solar information and still yield interesting results.

The motivation for this is obvious: neutrino experiments are extremely difficult to perform and particular detection schemes may suffer from some systematic error previously not considered. We make no judgements here about the errors associated with any particular experiment. Instead we consider analyses where we do not include one class of experiment, either water-Cerenkov, gallium or chlorine, which we designate \$K, Ø1 and \&a respectively. However, because we are losing an experiment, it is impossible to analyze the data without some level of information regarding the solar model. Consequently, we perform the analysis only within the solar SM inspired region. The results for this analysis are shown in figures 5 (upper row: without SuperKamiokande data, middle row: without chlorine data, and lower row: without gallium data).

The SK case largely resembles the complete data set analysis, with some additional space allowed in the higher $\Delta m_{12}^{2}$ region. In contrast, the other two cases ( $G$ ra and $\left.\not 1\right)$ show considerable differences.

For the fa case, there is a strong preference for either small $\Delta m_{12}^{2}$ or large $\theta_{12}$ and $\theta_{13}$. For the $\varnothing 1$ case, for both large $\theta_{12}$ and $\theta_{13}$ we have the presence of large regions with large $\Delta m_{12}^{2}=10^{-4} \mathrm{eV}^{2}$, above the levelcrossing threshold, and with small $\Delta m_{12}^{2}$, in the non-adiabatic region. In either case, in a large portion of these regions matter enhancements are unimportant. That is, in the absence of one of these two classes of experiment, given sufficiently large angles, the solar neutrino problem can be resolved simply by vacuum oscillations alone! In such a case, new experiments, such as Borexino, would see an absence of energy dependence in the electron neutrino survival probability.

\section{Atmospheric and Solar Neutrinos: The Minimal Scheme}

The simplest picture for reconciling both solar and atmospheric neutrino fluxes via oscillations of $\nu_{e, \mu, \tau}$ results when there is a hierarchy $\left|\Delta m_{23}^{2}\right| \gg\left|\Delta m_{12}^{2}\right|$, and $\Delta m_{12}^{2}$ is too small to affect oscillations of atmospheric neutrinos. In section 2, we showed that in this case the solar fluxes depend only on $\Delta m_{12}^{2}, \theta_{12}$ and $\theta_{13}$, and below we show that the atmospheric fluxes depend only on $\Delta m_{23}^{2}, \theta_{23}$ and $\theta_{13}$. In the limit that $\theta_{13}=0$, the two phenomena become independent, in the sense that they depend on no common parameters: solar oscillations are $\nu_{e} \rightarrow \nu_{\mu}$ at a low frequency, while atmospheric oscillations are $\nu_{\mu} \rightarrow \nu_{\tau}$ at a much higher frequency. However, solar oscillations are allowed for a wide range of parameters with large $\theta_{13}$, and the atmospheric data does not require $\theta_{13}$ to be very small. Hence, in this section we explore this simple picture keeping $\theta_{13}$ as a free parameter. We comment on the alternative possibility - that $\Delta m_{12}^{2}$ is large enough to contribute to atmospheric neutrino oscillations - in section 1 .

Matter effects in the earth are important only for a relatively small fraction of the atmospheric neutrinos, those with high energy, and they are neglected here ${ }^{3}$. In this case, (2.3) can be integrated to give oscillation probabilities $P_{f f^{\prime}}(t)=\left|A_{f f^{\prime}}(t)\right|^{2}$, where $A$ is given by the matrix equation

$$
A(t)=V e^{-i E t} V^{\dagger} .
$$

Since an overall phase in $A$ is irrelevant to $P$, and $\Delta m_{12}^{2}$ effects are negligible, we may make the substitution

$$
e^{-i E t} \longrightarrow \operatorname{diag}\left(1,1, e^{-i \Delta m_{23}^{2} t / 2 E}\right)
$$

Using the form (2.4) for $V$, we immediately discover that the probabilities are independent of $\theta_{12}$ and $\phi$, as well as $\alpha$ and $\beta$. The probabilities are given by

$$
\begin{aligned}
& P_{e \mu}=s_{23}^{2} \sin ^{2} 2 \theta_{13} S_{23} \\
& P_{e \tau}=c_{23}^{2} \sin ^{2} 2 \theta_{13} S_{23} \\
& P_{\mu \tau}=c_{13}^{4} \sin ^{2} 2 \theta_{23} S_{23}
\end{aligned}
$$

or equivalently, by unitarity

$$
\begin{aligned}
P_{e e} & =1-\sin ^{2} 2 \theta_{13} S_{23} \\
P_{\mu \mu} & =1-4 c_{13}^{2} s_{23}^{2}\left(1-c_{13}^{2} s_{23}^{2}\right) S_{23} \\
P_{\tau \tau} & =1-4 c_{13}^{2} c_{23}^{2}\left(1-c_{13}^{2} c_{23}^{2}\right) S_{23}
\end{aligned}
$$

\footnotetext{
${ }^{2}$ Although for non-zero $\theta_{13}$, there is a dependence on $\Delta m_{23}^{2}$ if it is small enough.

${ }^{3}$ For more details see e.g. ref. [17].
} 
where $S_{23}=\sin ^{2}\left(\Delta m_{23}^{2} t / 4 E\right)$. The parameter $\Delta m_{23}^{2}$ can be extracted from the data by fitting to the zenith angle distribution of the events. Here we concentrate on the determination of the parameters $\theta_{13}$ and $\theta_{23}$. These can be extracted, independent of the value of $\Delta m_{23}^{2}$, if we assume that the downward going neutrinos have not oscillated, while the upward going neutrinos are completely oscillated, so that $S_{23}$ is averaged to 0.5 . In view of the reported angular distribution of the multi-GeV data for 1-ring $e$-like, 1-ring $\mu$-like and partially contained (PC) events [3], this assumption appears to be valid, at least for angular cone sizes about the vertical which are not too large. For events of class $i$, which are induced by $\nu_{e}$ charged current, $\nu_{\mu}$ charged current and neutral current interactions with relative probabilities $f_{e C C}^{i}, f_{\mu C C}^{i}$ and $f_{N C}^{i}$, the up-down ratio $\rho_{i}$ is given by

$$
\rho_{i}=\frac{N_{i}^{\uparrow}}{N_{i}^{\downarrow}}=f_{e C C}^{i} \cdot\left(P_{e e}+r P_{e \mu}\right)+f_{\mu C C}^{i} \cdot\left(P_{\mu \mu}+\frac{1}{r} P_{e \mu}\right)+f_{N C}^{i}
$$

where we have set $S_{23}=0.5$, and $N_{i}^{\uparrow, \downarrow}$ are the number of upward and downward events of class $i$. We are interested in $i$ being 1-ring $e$-like, 1-ring $\mu$-like and PC. The overall normalization of these event numbers has considerable uncertainties due to the calculation of the neutrino fluxes produced in cosmic ray showers, hence we consider three up-down ratios

$$
\begin{aligned}
\rho_{e} & =1.23 \pm 0.29 \\
\rho_{\mu} & =0.62 \pm 0.16 \\
\rho_{P C} & =0.48 \pm 0.12
\end{aligned}
$$

and two ratios of downward going fluxes

$$
\begin{aligned}
\frac{N_{\mu}^{\downarrow}+N_{P C}^{\downarrow}}{N_{e}^{\downarrow}}=\xi r & =3.0 \pm 0.6 \\
\frac{N_{P C}^{\downarrow}}{N_{\mu}^{\downarrow}}=\xi^{\prime} & =1.3 \pm 0.3
\end{aligned}
$$

where $r$ is the ratio of $\nu_{\mu}$ to $\nu_{e}$ fluxes. The numbers give the Super-Kamiokande data, extracted from the figures of Ref. [3], with upward and downward directions defined by the azimuthal angle having $\cos \theta$ within 0.4 of the vertical direction. The parameters $\xi r$ and $\xi^{\prime}$ represent the theoretical values for the ratios of (3.5d) and (3.5e). These two downward going ratios do not involve oscillations, and the Super-Kamiokande collaboration compute Monte Carlo values of 3.1 and 1.0, respectively, agreeing very well with the data. Since these two ratios do not probe oscillations, at least within our assumptions, we do not use them for the fits below. We do not use the sub-GeV data as the poor angular correlation between the neutrino and charged lepton directions leads to a smoothing of the up-down ratio. From the flux calculations of Honda et al [18], and using the measured momentum distributions for the events [3], we estimate $r=4.0 \pm 0.5$, for this multi-GeV data near the vertical direction. A more refined analysis would use a larger value of $r$ for PC events than for FC events.

The results of a fit of the three up/down ratios to the two free parameters $\theta_{23}$ and $\theta_{13}$ are shown in figure 6(a). We have obtained the fractions $f_{e C C, \mu C C, N C}^{i}$ from the Monte Carlo results of the Super-Kamiokande collaboration [3], and we have used the oscillation probabilities of (3.3c). In order to work with Gaussian distributed experimental data, we have directly fitted the six measured neutrino numbers $N_{i}^{\uparrow, \downarrow}$ leaving arbitrary the three overall fluxes of each type, $N_{i}^{\uparrow}+N_{i}^{\downarrow}$. The preferred region of the plot is easy to understand, since at the point $\theta_{23}=45^{\circ}$ and $\theta_{13}=0$, the $\nu_{e}$ are unmixed, while there is complete $\nu_{\mu} \leftrightarrow \nu_{\tau}$ mixing, so $\rho_{e} \simeq 1$ and $\rho_{\mu} \simeq \rho_{P C} \simeq 0.5$. It is apparent from Fig. 6(a) that this minimal scheme is allowed for a large range of angles about this point: $\theta_{23}=45^{\circ} \pm 15^{\circ}$ and $\theta_{13}=0 \div 45^{\circ}$.

If the solar neutrino fluxes, measured by all three techniques, are to agree with solar model inspired values, then the results of section 2 show that $\Delta m_{12}^{2}$ is too small to affect atmospheric oscillations, it is either of order $10^{-4}-10^{-5} \mathrm{eV}^{2}$ or of order $10^{-10} \mathrm{eV}^{2}$. In this case, the minimal scheme for atmospheric neutrinos, described in this section, is the unique possibility using just the three known neutrinos. This observation enhances the importance of the fit of figure 6(a); further data will reduce the allowed region, as the three up-down ratios of (3.5c) have small systematic uncertainties and are statistics limited. The solar neutrino fluxes do not put extra constraints on the value of $\theta_{13}$, although it becomes correlated with $\theta_{12}$, as shown in figure 3. If the atmospheric flux measurements require $\Delta m_{23}^{2}>2 \times 10^{-3} \mathrm{eV}^{2}$, then the limit on $P_{e e}$ from the CHOOZ experiment [19] requires $\theta_{13}<13^{\circ}$.

Recent analyses [20] of SuperKamiokande data that make use of MonteCarlo predictions for the angular and energy distributions of the atmospheric neutrinos get more stringent constraints on the neutrino oscillation parameters. Our fit uses only those data - the ratio of upward and downward multi-GeV neutrinos (the ones 
(a)

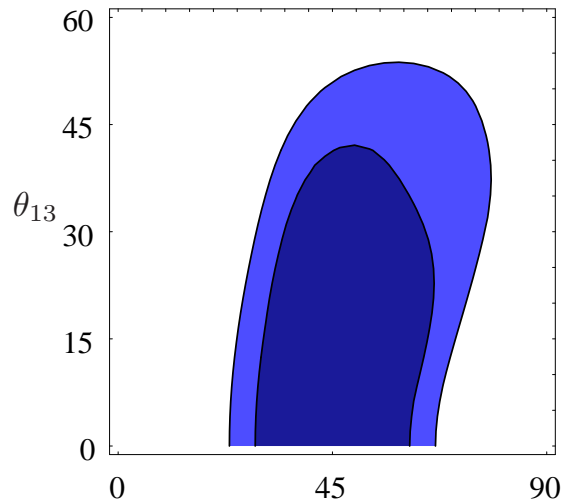

(a')

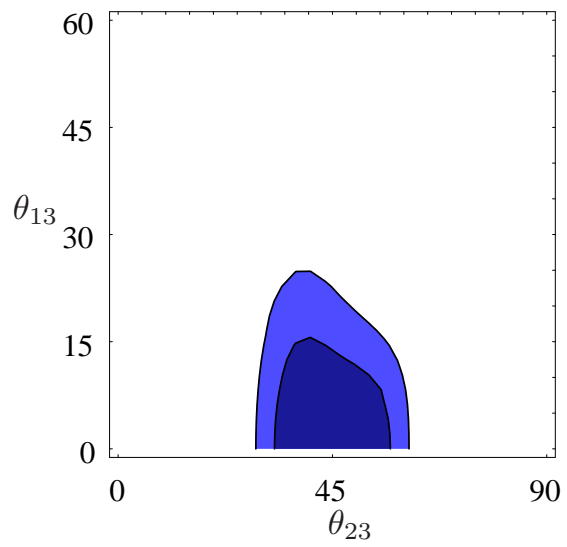

(b1)

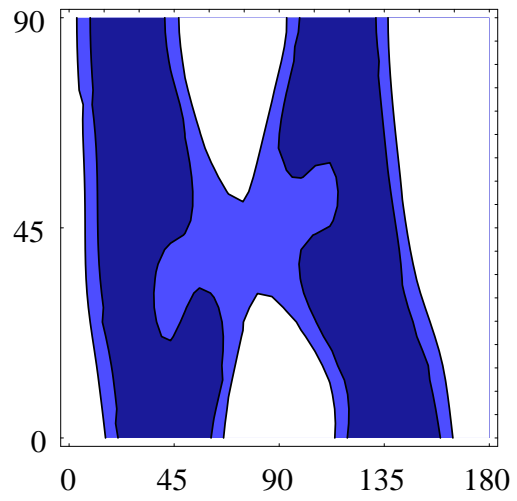

(b1')

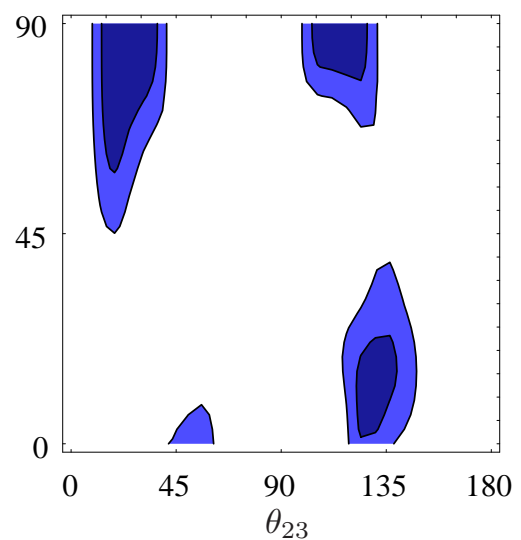

(b2)

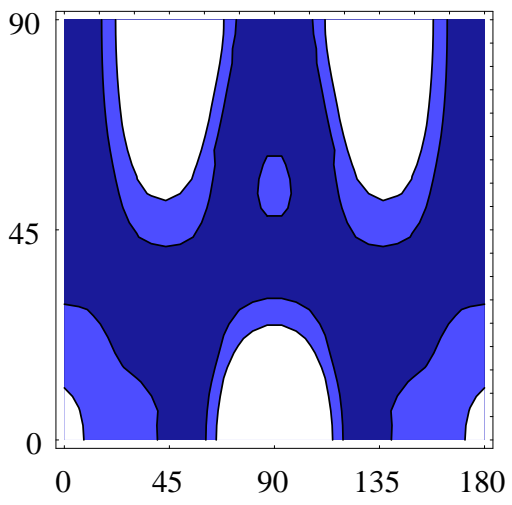

(b2')

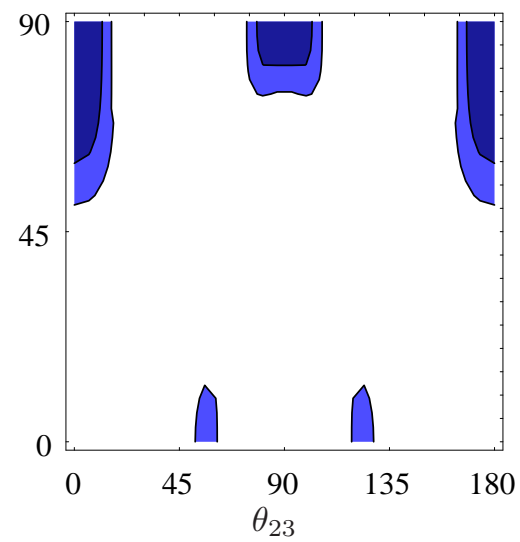

Figure 6: Mixing angles $\theta_{i j}$ that fit the up/down ratios (3.5a,b,c) of atmospheric neutrinos, assuming that (a) $\Delta m_{12}^{2} \ll 10^{-3} \mathrm{eV}^{2}$ and any $\theta_{12}$, (b) $\Delta m_{12}^{2} \approx \Delta m_{23}^{2} \approx 10^{-3} \mathrm{eV}^{2}, \phi=0$ and (b1) $\theta_{12}=20^{\circ}$, (b2) $\theta_{12}=45^{\circ}$. Primed figures are as above, but including in the asymmetry also the intermediate bins in the angular distribution of [3] (see text). The contours are for $\chi^{2}=3$ and $\chi^{2}=6$.

in bins 1 and 5 of the angular distribution in [3]) - that do not depend on the spectrum of the atmospheric neutrinos nor on the precise value of $\Delta m^{2}$, assuming a full averaged oscillation in between. Since statistics gives presently the dominant error, we obtain weaker constraints than in 20]. If we knew that the neutrino mass difference relevant for atmospheric neutrinos were close to the center of the presently allowed region, we could add to the data to be fitted the intermediate bins 2 and 4 of [3] (the bins that contain 'oblique' neutrinos). We cannot use in any case the multi-GeV data in the intermediate bin 3, that contains 'horizontal' neutrinos. Having doubled the statistics, we would find the more stringent contours shown in fig. 6a', b1', b2'. We remind the reader that our fit does not include matter effects [1].

\section{Atmospheric and Solar Neutrinos: Non-Minimal Schemes}

In this section, we study atmospheric neutrinos when two conditions apply.

- The smallest mass splitting is large enough to affect atmospheric neutrino oscillations: $\Delta m_{12}^{2}>3 \times$ $10^{-4} \mathrm{eV}^{2}$. For solar neutrinos, this implies that there is a serious flaw either in at least one measurement technique, or in the solar models.

- The mass splittings are hierarchical $\Delta m_{23}^{2} \gg \Delta m_{12}^{2}$. This is a simplification, which we relax at the end of the section. It includes the interesting possibility that $\Delta m_{23}^{2}$ is large enough to induce the apparent oscillations reported by the LSND collaboration [21], while $\Delta m_{12}^{2}$ effects are causing both solar and atmospheric oscillations.

Using (2.5), the $\nu_{e}$ survival probability is

$$
P_{e e}=1-c_{13}^{4} \sin ^{2} 2 \theta_{12} S_{12}-s_{12}^{2} \sin ^{2} 2 \theta_{13} S_{23}-c_{12}^{2} \sin ^{2} 2 \theta_{13} S_{31}
$$


where $S_{i j}=\sin ^{2}\left(\Delta m_{i j}^{2} t / 4 E\right)$. The above two conditions imply that $\Delta m_{23}^{2}>2 \times 10^{-3} \mathrm{eV}^{2}$, so that, for the CHOOZ experiment, (4.1) should be used with $S_{23}=S_{31}=0.5$. The CHOOZ limit, $P_{e e}>0.9$, then gives $\theta_{13}<0.23$. If $\Delta m_{12}^{2}$ were also greater than $2 \times 10^{-3} \mathrm{eV}^{2}$, then for the CHOOZ experiment one also has $S_{12}=0.5$, so that $\theta_{12}<0.23$. However, in this case the survival probability for solar neutrinos is the same as for the anti-neutrinos at CHOOZ: $P_{e e}>0.9$. Hence, given our two conditions, the observed solar neutrino fluxes require $\Delta m_{12}^{2}<2 \times 10^{-3} \mathrm{eV}^{2}$.

It is frequently stated that the three known neutrinos cannot explain the LSND, atmospheric and solar neutrino anomalies, as this would require three $\Delta m^{2}$ with different orders of magnitudes. However, this argument no longer applies in the case that either a solar neutrino measurement technique or solar models are incorrect, when a single $\Delta m^{2}$ could give both atmospheric and solar anomalies. Hence, we consider first the case that $\Delta m_{23}^{2}$ is large enough to explain the observations of LSND. The oscillation probabilities induced by $\Delta m_{23}^{2}$ are given by (3.3c). From the limit on $P_{e e}$ from the Bugey reactor, one then concludes

$$
\Delta m_{23}^{2}>0.2 \mathrm{eV}^{2}
$$

and

$$
\theta_{13}<0.1
$$

which is significantly stronger than the CHOOZ limit. A second possibility, $\theta_{13}$ close to $90^{\circ}$ does not allow any significant oscillations of $\nu_{e}$ and is thus not acceptable to explain the solar neutrino anomaly at a relatively large frequency. For atmospheric neutrinos, both upward going and downward going, one may then use oscillation probabilities with $\theta_{13}=0$ 用 and with $S_{23}$ and $S_{13}$ both averaged to 0.5:

$$
\begin{aligned}
& P_{e \mu}=c_{23}^{2} \sin ^{2} 2 \theta_{12} S_{12} \\
& P_{e \tau}=s_{23}^{2} \sin ^{2} 2 \theta_{12} S_{12} \\
& P_{\mu \tau}=-\frac{1}{4} \sin ^{2} 2 \theta_{23} \sin ^{2} 2 \theta_{12} S_{12}+\frac{1}{2} \sin ^{2} 2 \theta_{23}
\end{aligned}
$$

or equivalently, from unitarity

$$
\begin{aligned}
P_{e e} & =1-\sin ^{2} 2 \theta_{12} S_{12} \\
P_{\mu \mu} & =1-\frac{1}{2} \sin ^{2} 2 \theta_{23}-c_{23}^{4} \sin ^{2} 2 \theta_{12} S_{12} \\
P_{\tau \tau} & =1-\frac{1}{2} \sin ^{2} 2 \theta_{23}-s_{23}^{4} \sin ^{2} 2 \theta_{12} S_{12} .
\end{aligned}
$$

Since in these formulæ $S_{13}=S_{23}, \theta_{12}$ enters only via $\sin ^{2} 2 \theta_{12}$ so that, without loss of generality, we may reduce the range of $\theta_{12}$ to $0 \leq \theta_{12}<\pi / 4$. We again study the up-down ratios (3.4), as they have small systematic uncertainties. We calculate them approximately, using (3.4) with $f_{e C C}^{e}=f_{\mu C C}^{\mu}=f_{\mu C C}^{P C}=1$ and all other $f$-factors equal to zero. A fraction, $P_{\mu \mu}^{(0)}=1-\sin ^{2} 2 \theta_{23} / 2$, of the downward going $\nu_{\mu}$ oscillate to $\nu_{\tau}$ before detection, so the up-down ratios are given by

$$
\rho_{e} \approx P_{e e}+r P_{e \mu}
$$

and

$$
\rho_{\mu} \approx \frac{P_{\mu \mu}+P_{e \mu} / r}{P_{\mu \mu}^{(0)}}
$$

Hence we find

$$
\left(\rho_{\mu}-1\right) \approx-\frac{1}{r} \frac{c_{23}^{2}}{1-\frac{1}{2} \sin ^{2} 2 \theta_{23}} \cdot\left(\rho_{e}-1\right) .
$$

For the multi-GeV data, where the angular correlation is best, $r$ is large, and (4.6) implies that $\left|\rho_{\mu}-1\right|<$ $(1 / 3)\left|\rho_{e}-1\right|$, in strong disagreement with data of (3.5c). The same inequality holds if $\rho_{\mu}$ is replaced by $\rho_{P C}$, when the disagreement with data is even stronger.5 With oscillations of the three known neutrinos, the LSND observation conflicts with the atmospheric and solar neutrino anomalies even using a model independent analysis of the solar neutrino fluxes or allowing for a systematic error in one of the solar neutrino experiments 9 .

Does the atmospheric neutrino data allow other values of $\Delta m_{23}^{2} \gg \Delta m_{12}^{2}$ ? The limit from the Bugey reactor, (4.2b), applies for all $\Delta m_{23}^{2}>0.06 \mathrm{eV}^{2}$, and the up-down ratio relation, (4.6), applies for all $\Delta m_{23}^{2}>0.1 \mathrm{eV}^{2}$.

\footnotetext{
${ }^{4}$ In which case the $P_{i j}$ are independent of $\phi$.

${ }^{5}$ Even ignoring $\rho_{e}$, we find $\rho_{P C, \mu}>0.61$.

${ }^{6}$ For $\theta_{23}=0$, this corresponds to purely $\nu_{\mu} \rightarrow \nu_{e}$ oscillations, which is therefore excluded as an explanation of the atmospheric neutrino measurements.
} 
Hence, $\Delta m_{23}^{2}>0.1 \mathrm{eV}^{2}$ is excluded. For $\Delta m_{23}^{2}<0.1 \mathrm{eV}^{2}$, the downward going $\nu_{\mu}$ have not oscillated to $\nu_{\tau}$ when they reach the Super-Kamiokande detector, so that (4.5) is replaced by

$$
\rho_{\mu} \approx P_{\mu \mu}+\frac{1}{r} P_{e \mu}=1-\frac{1}{2} \sin ^{2} 2 \theta_{23}-\frac{c_{23}^{2}}{r}\left(\rho_{e}-1\right) .
$$

Consistency with the data, (3.5c), is now possible, and requires large $\theta_{23}$. As $\Delta m_{23}^{2}$ drops below $0.06 \mathrm{eV}^{2}$, the limit from the Bugey reactor on $\theta_{13}$ is progressively weakened, so that $\theta_{13}$ terms must be kept in $P_{i j}$. Furthermore, as $\Delta m_{23}^{2}$ drops below $0.01 \mathrm{eV}^{2}$, our hierarchy condition is no longer satisfied, so that $P_{i j}$ depend also on $\theta_{12}$. For these cases we have performed a $\chi$ squared fit of the three up-down ratios (3.5c) to $\theta_{23}, \theta_{13}$ and $\theta_{12}$, for various values of the mass splittings, and have found acceptable regions of parameter space. Results are shown in figure $6 \mathrm{~b}$ for the case that all $S_{i j}=0$ for downward going neutrinos, while all $S_{i j}=0.5$ for upward going neutrinos and $\phi=0$ (no CP violation). An equivalent fit would be obtained for $\phi=\pi$ and $\theta_{23} \rightarrow \pi-\theta_{23}$. The (relatively small) asymmetry of fig.s $6 \mathrm{~b}$ under $\theta_{23} \rightarrow \pi-\theta_{23}$ shows the dependence on $\phi$ of the SuperKamiokande data considered here.

A comparison of figure $6 \mathrm{~b}$ with figures 3 and 5 shows under what conditions this large $\Delta m_{12}^{2}$ scheme gives consistency. If all solar measurement techniques are correct, then, from figure 3, $\theta_{13}$ is small and $\theta_{12}=10^{\circ} \div 20^{\circ}$. Figure $6 \mathrm{~b}$ then shows that $\theta_{23}$ is centred on $45^{\circ} \pm 25^{\circ}$, the range around $\theta_{23}=135^{\circ}$ being equivalent for any $\phi$ since $\theta_{13}$ is small. Figure 5 shows that solar model inspired fits to data from two solar techniques at large $\Delta m_{12}^{2}$ allow larger ranges of $\theta_{12}$ and $\theta_{13}$, and these become correlated with $\theta_{23}$ via figure Gb.

\section{$5 \quad$ Large $\nu_{\mu} \rightarrow \nu_{\tau}$ Mixing For Atmospheric Neutrinos}

The pattern of masses and mixings suggested by the previous considerations show peculiar features, especially if both the atmospheric and solar neutrino anomalies are accounted for in the minimal scheme of section 3 . The mass differences are hierarchical. However a large mixing $\left(\theta_{23} \approx 45^{\circ}\right)$ is required between the states with the largest mass difference. The mixing angle $\theta_{12}$ between the states with the smallest mass splitting may be large or small. Finally, if $\Delta m_{\mathrm{atm}}^{2} \geq 2 \cdot 10^{-3} \mathrm{eV}^{2}$, i.e. in the CHOOZ range, the third mixing angle must be small, $\theta_{13} \leq 13^{\circ}$. Therefore it looks interesting to see which mass matrix could produce this pattern and which flavour symmetries can justify it.

\section{$5.12 \times 2$ Matrix Forms}

As stressed in the introduction, an important consequence of the data on atmospheric neutrino fluxes is the need for large mixing angles. Here we study four possible forms of the $2 \times 2$ Majorana mass matrix for $\nu_{\mu}$ and $\nu_{\tau}$ which have a large mixing angle. In subsection 5.2 we study whether these forms can be incorporated in $3 \times 3$ mixing schemes which also give solar neutrino oscillations, and whether $3 \times 3$ cases exist which cannot be reduced to a $2 \times 2$ form. In section 6 we study whether these forms may be obtained from flavour symmetries of abelian type.

In a basis with a diagonal charged lepton mass matrix, the Majorana neutrino mass matrix is

$$
m=\frac{v^{2}}{M}\left(\begin{array}{cc}
C & B \\
B & A
\end{array}\right) .
$$

This is brought into real, diagonal form by the unitary matrix

$$
V=R(\theta)\left(\begin{array}{cc}
1 & 0 \\
0 & e^{i \alpha}
\end{array}\right)
$$

where $\tan 2 \theta=2 B /(A-C)$, and the phase $\alpha$ does not affect oscillations. The mass difference relevant for oscillations is $\Delta m^{2}=(A+C) \sqrt{(A-C)^{2}+4 B^{2}}$. The coefficient $v^{2} / M$ is motivated by the see-saw mechanism, with $v$ the electroweak vacuum expectation value and $M$ the mass of a heavy right-handed neutrino.

There are four possible forms of this matrix which give $\theta \approx 1$, and these are shown in Table 1 . In cases (1) and (2) the entries are all of order unity; in the generic case they are unrelated, while in case (2) they are related in such a way that the determinant is suppressed. We discuss how such a suppression can occur naturally via the seesaw mechanism in the next section. Case (3) has one of the diagonal entries suppressed, which, however, does not follow from a simple symmetry argument. For cases $(1 \div 3)$, taking $\Delta m^{2}=10^{-3} \mathrm{eV}^{2}$, one finds

$$
M=(1 \div 3) \times 10^{15} \mathrm{GeV}
$$




\begin{tabular}{|l|c|cll|}
\hline & small entries & small parameters & order unity parameters & $\Delta m^{2} /\left(\frac{v^{2}}{M}\right)^{2}$ \\
\hline$(1)$ Generic & none & none & $A, B, C$ & $\approx 1$ \\
$(2)$ Determinant small & none & none & $A, B, C=B^{2} / A+\varepsilon$ & $\approx 1$ \\
$(3)$ One diagonal small & one diagonal & $C \approx \varepsilon$ & $A, B$ & $\approx 1$ \\
$(4)$ Pseudo-Dirac & both diagonal & $A, C \approx \varepsilon$ & $B$ & $\approx \varepsilon$ \\
\hline
\end{tabular}

Table 1: The four possible $2 \times 2$ matrix forms which give a large mixing angle.

close to the scale of gauge coupling unification in supersymmetric theories.

Finally, case (4) has both diagonal entries small, making $\nu_{\mu}$ and $\nu_{\tau}$ components of a pseudo-Dirac neutrino. This follows from an approximate $L_{\mu}-L_{\tau}$ symmetry, and implies that $\theta \simeq 45^{\circ}$. This agrees well with data: combing $\rho_{\mu}$ and $\rho_{P C}$ of (3.5 ) gives $\theta=45^{\circ} \pm 15^{\circ}$. Of the four possible cases with large mixing angle, it is only the pseudo-Dirac neutrino which allows $\nu_{\mu, \tau}$ to be the astrophysical hot dark matter, in which case one predicts $\theta=45^{\circ}$ to high accuracy.

From the viewpoint of atmospheric neutrino oscillations alone, the distinction between cases (1) and (2) is unimportant. Since case (3) does not follow from simple symmetry arguments, one is left with two main $2 \times 2$ mixing schemes: the generic and pseudo-Dirac cases.

\section{$5.23 \times 3$ Matrix Forms}

There are many possibilities for $3 \times 3$ neutrino mixing giving $P_{\mu \mu} \approx 0.5$, with oscillation primarily to $\nu_{\tau}$. In general two independent frequencies and three Euler angles are involved.

For the case that the oscillation is dominated by a single frequency, the possibilities may be divided into two classes: " $2 \times 2$-like" and "inherently $3 \times 3$." The $2 \times 2$-like cases are just the four discussed in subsection 5.1, with $\theta_{12,13}$ small. Even though $\Delta m_{23}^{2}$ may not be the largest $\Delta m^{2}$, it is the only one which causes substantial depletion of $\nu_{\mu}$. More interesting are the inherently $3 \times 3$ cases, for which there is no $2 \times 2$ reduction.

Consider the case

$$
m=\frac{v^{2}}{M}\left(\begin{array}{ccc}
0 & B & A \\
B & 0 & 0 \\
A & 0 & 0
\end{array}\right)
$$

with $A, B \approx 1$. This is diagonalized by $V=R_{23}\left(\theta_{23}\right) R_{12}\left(\theta_{12}=45^{\circ}\right)$ giving a Dirac state of $\nu_{e}$ married to $c_{23} \nu_{\mu}+s_{23} \nu_{\tau}$. The mass eigenvalues are $(M, M, 0)$, which, from the viewpoint of oscillations are equivalent to $(0,0, M)$. Hence, one immediately sees that the oscillation probabilities are given by (3.3c with $\theta_{13}=0$ : $P_{\mu \tau}=\sin ^{2} 2 \theta_{23} S_{23}$ has the form of a $2 \times 2$ oscillation, even though the mass matrix has an inherently $3 \times 3$ form. This arises because (5.4) is governed by the symmetry $L_{e}-L_{\mu}-L_{\tau}$, which allows $\nu_{\mu} \leftrightarrow \nu_{\tau}$, but prevents $\nu_{e}$ from oscillating.

We claim that (5.4) is the only inherently $3 \times 3$ form for $\nu_{\mu} \rightarrow \nu_{\tau}$ at a single frequency, as we now show. An inherently $3 \times 3$ form must have large entries outside the $2 \times 2$ block in 23 subspace. The three possibilities are 11, 12 and 13 (and their symmetric). None of these entries work alone, even coupled to any structure in the 23 block: either one gets two comparable frequencies or one does not get $\nu_{\mu} \rightarrow \nu_{\tau}$. The same is true for $11+12$ or $11+13$, again possibly together with any 23 -block. Since $11+12+13$ leads to two comparable frequencies, the only case remaining is $12+13$, with a relatively negligible 23 block, i.e. the $3 \times 3$ form in (5.4). Basic to this conclusion is the assumption of no special relations among the different neutrino matrix elements other than the symmetry of the matrix itself (for alternatives see [22]).

\section{Models for both Solar and Atmospheric Neutrinos}

In this section we construct models for the minimal scheme for atmospheric and solar neutrino oscillations, discussed in section 3. The mass pattern suggested by this scheme has the hierarchy $\Delta m_{\odot}^{2} \equiv \Delta m_{12}^{2} \ll \Delta m_{\text {atm }}^{2} \equiv$ $\Delta m_{23}^{2}$. We take the form of the lepton mass matrices to be determined by flavour symmetries (FS) and assume that all small entries in these matrices are governed by small flavour symmetry breaking (FSB) parameters.

The low energy effective mass matrix for the three light left-handed neutrinos can be written as the sum of two matrices: $m_{L L}=m_{\mathrm{atm}}+m_{\odot}$, where all non-zero entries of $m_{\mathrm{atm}}$ are larger than all entries of $m_{\odot}$. 
The form of $m_{\text {atm }}$ is such that there is a large mass splitting: $\Delta m_{\text {atm }}^{2} \approx 10^{-(2 \div 3)} \mathrm{eV}^{2}$, and a vanishing $\Delta m^{2}$. Furthermore, this matrix must give a large depletion of $\nu_{\mu}$, and, as discussed in the last section, this could occur if it has certain $2 \times 2$-like or inherently $3 \times 3$ forms. Of the two $2 \times 2$-like forms shown in Table 1 , only case (2) is acceptable: in cases (1) and (3) the two independent $\Delta m^{2}$ are comparable, while in case (4) the second independent $\Delta m^{2}$ is larger than $\Delta m_{\text {atm }}^{2}$. Hence, we arrive at the possibility:

$$
m_{\mathrm{atm}}^{2 \times 2}=\frac{v^{2}}{M}\left(\begin{array}{ccc}
0 & 0 & 0 \\
0 & C & B \\
0 & B & A
\end{array}\right)
$$

with $A, B \approx 1$ and $C=B^{2} / A$. A reason for the vanishing sub-determinant will be given shortly.

In the previous section we have proved that there is a unique form for $m_{\text {atm }}$ which is inherently $3 \times 3$ :

$$
m_{\mathrm{atm}}^{3 \times 3}=\frac{v^{2}}{M}\left(\begin{array}{ccc}
0 & B & A \\
B & 0 & 0 \\
A & 0 & 0
\end{array}\right)
$$

with $A, B \approx 1$.

The oscillation angles in the leptonic mixing matrix, $V$, have contributions from diagonalization of both the neutrino mass matrix, $\theta_{i j}^{\nu}$, and the charged lepton mass matrix, $\theta_{i j}^{e}: V\left(\theta_{i j}\right)=V^{e \dagger}\left(\theta_{i j}^{e}\right) V^{\nu}\left(\theta_{i j}^{\nu}\right)$. This requires discussing also the charged lepton mass matrix. It is not easy to construct an exhaustive list of the possible symmetries and their breaking parameters. This is partly because there are both discrete and continuous symmetries with many choices for breaking parameters; but is mainly because of a subtlety of the seesaw mechanism. Let $m_{R R}$ and $m_{L R}$ be the most general Majorana and Dirac mass matrices of the seesaw mechanism allowed by some approximate symmetry. On forming the mass matrix for the light states, $m_{L L}=m_{L R} m_{R R}^{-1} m_{L R}^{T}$, one discovers that $m_{L L}$ need not be the most general matrix allowed by the approximate symmetry. This means that one cannot construct an exhaustive list by only studying the symmetry properties of $m_{L L}$ - it is necessary to study the full theory containing the right-handed states.

A casual glance at (6.1) and (6.2) shows that the flavor symmetry we seek, from the viewpoint of $\Delta L=2$ operators, does not distinguish $l_{\mu}$ from $l_{\tau}$, but does distinguish these from $l_{e}$. There are many combinations of the three lepton numbers $L_{a}$, and their subgroups, acting on $l_{a}$, which have this property. As representative of this group, we choose the combination $L_{e}-L_{\mu}-L_{\tau}$. We find it remarkable that this symmetry group can yield both (6.1) and (6.2), depending on how it is realized.

\section{1 $L_{e}-L_{\mu}-L_{\tau}$ realized in the Low Energy Effective Theory}

In the effective theory at the weak scale, we impose an approximate $L_{e}-L_{\mu}-L_{\tau}$ symmetry, which acts on the weak doublets, $l_{e, \mu, \tau}$, and is broken by small FSB parameters, $\varepsilon$ and $\varepsilon^{\prime}$ of charge +2 and -2 , respectively, giving a neutrino mass matrix:

$$
m_{L L}=\frac{v^{2}}{M}\left(\begin{array}{lll}
\varepsilon^{\prime} & 1 & 1 \\
1 & \varepsilon & \varepsilon \\
1 & \varepsilon & \varepsilon
\end{array}\right)
$$

Hereafter, the various entries of the matrices only indicate the corresponding order of magnitude, allowing for an independent parameter for each entry. This texture gives

and

$$
\begin{aligned}
& m_{1} \approx m_{2} \approx \frac{v^{2}}{M} \quad \Delta m_{12}^{2} \approx \frac{v^{4}}{M^{2}}\left(\varepsilon+\varepsilon^{\prime}\right) \\
& m_{3} \approx \frac{v^{2}}{M} \varepsilon \quad \Delta m_{23}^{2} \approx \frac{v^{4}}{M^{2}}
\end{aligned}
$$

$$
\theta_{23}^{\nu} \approx 1 \quad \theta_{13}^{\nu} \approx \varepsilon \quad \theta_{12}^{\nu}=45^{\circ} .
$$

While the texture gives only the order of magnitude of $\theta_{23}^{\nu}$, it precisely predicts $\theta_{12}^{\nu}$ to be close to $45^{\circ}$. If the FSB parameters $\varepsilon$ and $\varepsilon^{\prime}$ are taken to be extremely small, this becomes an excellent candidate for the case of "just so" solar neutrino oscillations, with the prediction that $\theta_{12}=45^{\circ}$. However, from figure 3 it follows that this model cannot give matter neutrino oscillations in the sun, which requires $\sin 2 \theta_{12} \leq 0.9$. There are several

\footnotetext{
${ }^{7}$ Ansätze of this type for the neutrino mass matrix, up to small corrections, to describe atmospheric and solar neutrinos are contained in ref.s [23].
} 
contributions to the deviation of $\sin 2 \theta_{12}$ from unity, but they are all too small to reconcile the discrepancy. A hierarchy in $\Delta m^{2}$ requires $\varepsilon, \varepsilon^{\prime}<0.1$, and since $\sin ^{2} 2 \theta_{12}^{\nu} \simeq 1-\left(\varepsilon-\varepsilon^{\prime}\right)^{2} / 8$, the deviation of $\sin 2 \theta_{12}^{\nu}$ from 1 is negligible. After performing the $\theta_{12}^{\nu}$ rotation, there are small $\mathcal{O}(\varepsilon)$ rotations in the 13 and 23 planes necessary to fully diagonalize $m_{L L}$; these are too small to affect our conclusions. The last hope is that there could be a significant contribution to $\theta_{12}$ from diagonalization of the charged lepton mass matrix. As mentioned above, the diagonalization of the charged lepton mass matrix has to be discussed anyhow.

Consistently with the symmetry structure of (6.3), the most general form for the charged lepton mass matrix, with a structure governed by abelian symmetries is

$$
m_{E}=\lambda v\left(\begin{array}{lll}
\xi^{\prime} & \xi \varepsilon^{\prime} & \varepsilon^{\prime} \\
\xi^{\prime} \varepsilon & \xi & 1 \\
\xi^{\prime} \varepsilon & \xi & 1
\end{array}\right)
$$

when left (right) handed leptons are contracted to the left (right), $\bar{e}_{L} m_{E} e_{R}$. $\left(1, \xi, \xi^{\prime}\right)$ are the relative FSB parameters of $\left(\tau_{R}, \mu_{R}, e_{R}\right)$ with respect to some other approximate FS, needed to describe the charged lepton mass hierarchies, and $\lambda$ is the absolute FSB parameter of $\bar{\tau}_{R} \tau_{L}$. Here we ignore the fact that non-abelian symmetries could modify this form, for example by requiring some entries to vanish.

Diagonalization of (6.5) leads to

$$
\theta_{23}^{e} \approx 1, \quad \theta_{13}^{e} \approx \varepsilon^{\prime}, \quad \theta_{12}^{e} \approx \varepsilon^{\prime}
$$

Therefore, altogether

$$
\theta_{23} \approx 1, \quad \theta_{12} \approx \varepsilon+\varepsilon^{\prime}, \quad \theta_{12} \approx 45^{\circ} .
$$

Since $\sin ^{2} 2 \theta_{12}$ remains corrected only by quadratic terms in $\varepsilon$ and/or $\varepsilon^{\prime}$, we conclude that $L_{e}-L_{\mu}-L_{\tau}$, realized as an approximate symmetry of the low energy effective theory, can explain both atmospheric and solar neutrino fluxes with a hierarchy of $\Delta m^{2}$, most likely only for the case of "just so" vacuum solar oscillations, in which case the scale of new physics, $M$, is close to the gauge unification scale, and the FSB parameters are extremely small: $\varepsilon, \varepsilon^{\prime} \approx 10^{-7}$. This result also applies when any approximate FS of the low energy effective theory yields (6.3). In view of (6.4), with $\Delta m_{23}^{2} \approx \Delta m_{\mathrm{atm}}^{2} \approx 10^{-(2 \div 3)} \mathrm{eV}^{2}$, notice that all three neutrinos are cosmologically irrelevant. Furthermore, the smallness of the 11 entry of $(6.3)$ makes the search for neutrino-less $2 \beta$-decay uninteresting.

Comparing the $\theta_{13}$ plots of figures 3 and 5 , one finds that, with one experiment excluded, the case of $\theta_{12}=45^{\circ}$ becomes allowed for a large range of $\Delta m_{12}^{2}$, giving another application for this inherently $3 \times 3$ form of the mass matrix.

\section{2 $L_{e}-L_{\mu}-L_{\tau}$ realized via the Seesaw Mechanism}

The seesaw mechanism 24] allows a simple origin for the vanishing of the $2 \times 2$ sub-determinant of (6.1). Consider a single right-handed neutrino, $N$, with Majorana mass $M$ and Dirac mass term $v N\left(\cos \theta \nu_{\tau}+\sin \theta \nu_{\mu}\right)$, where $\theta \approx 1$. Integrating out this single heavy state produces a single non-zero eigenvalue in $m_{L L}$ - giving (6.1) with $A=\cos ^{2} \theta, B=\cos \theta \sin \theta$ and $C=\sin ^{2} \theta$, so that $A C=B^{2}$.

How could this carry over to a theory with three right-handed neutrinos, $N_{a}$ ? As long as one of them, $N$ with the above mass terms, is much lighter than the others, then it will give the dominant contribution to $m_{L L}$, which will have (6.1) as its leading term. Clearly the key is that there be one right-handed neutrino which is lighter than the others, and couples comparably to $\nu_{\mu}$ and $\nu_{\tau}$.

This can be realized using $L_{e}-L_{\mu}-L_{\tau}$, with two small FSB parameters $\varepsilon(+2)$ and $\varepsilon^{\prime}(-2)$. The right-handed neutrino mass matrix is

$$
m_{R R}=M\left(\begin{array}{ccc}
\varepsilon^{\prime} & 1 & 1 \\
1 & \varepsilon & \varepsilon \\
1 & \varepsilon & \varepsilon
\end{array}\right)
$$

and the Dirac mass matrices of neutrinos and charged leptons are

$$
m_{L R}=\lambda^{\prime} v\left(\begin{array}{ccc}
\eta^{\prime} & \varepsilon \eta^{\prime} & \varepsilon \eta^{\prime} \\
\varepsilon^{\prime} \eta & \eta & \eta \\
\varepsilon^{\prime} & 1 & 1
\end{array}\right) \quad \text { and } \quad m_{E}=\lambda v\left(\begin{array}{ccc}
\xi^{\prime} \eta^{\prime} & \varepsilon \xi \eta^{\prime} & \varepsilon \eta^{\prime} \\
\xi^{\prime} \varepsilon^{\prime} \eta & \xi \eta & \eta \\
\xi^{\prime} \varepsilon^{\prime} & \xi & 1
\end{array}\right)
$$

where, in analogy with (6.5), we have introduced FSB parameters consistent with 6.7). 
For ease of exposition, let us first consider the case where all the $\eta$ and $\xi$ factors are set equal to unity. The crucial point is that there is a massless right-handed neutrino in the limit $\varepsilon \rightarrow 0$. Hence, taking $\varepsilon$ small, and doing a rotation in the 23 plane we have $2 \times 2$ sub-matrices

$$
m_{R R}^{-1}=\frac{1}{M}\left(\begin{array}{cc}
0 & 0 \\
0 & 1 / \varepsilon
\end{array}\right), \quad m_{L R}=\lambda^{\prime} v\left(\begin{array}{ll}
1 & 1 \\
1 & 1
\end{array}\right)
$$

giving

$$
m_{L L}=\frac{\left(\lambda^{\prime} v\right)^{2}}{M}\left(\begin{array}{ll}
1 / \varepsilon & 1 / \varepsilon \\
1 / \varepsilon & 1 / \varepsilon
\end{array}\right)
$$

where det $m_{L L}=0$ at this order. In a theory with right-handed neutrinos, $L_{e}-L_{\mu}-L_{\tau}$ leads to (6.1).

Extending the analysis to $3 \times 3$ matrices is straightforward. The inverse of $m_{R R}$

$$
m_{R R}^{-1}=\frac{1}{M}\left(\begin{array}{ccc}
\varepsilon & 1 & 1 \\
1 & \varepsilon^{\prime} & \varepsilon^{\prime} \\
1 & \varepsilon^{\prime} & \frac{1}{\varepsilon}
\end{array}\right)
$$

shows a pseudo-Dirac structure in the 12 subspace, which is preserved in the light neutrino mass matrix:

$$
m_{L L}=\frac{\left(\lambda^{\prime} v\right)^{2}}{M}\left(\begin{array}{ccc}
\varepsilon & 1 & 1 \\
1 & \varepsilon^{\prime} & 0 \\
1 & 0 & \frac{1}{\varepsilon}
\end{array}\right)
$$

where we have gone to a basis which diagonalizes the 23 subspace. The parameters relevant for neutrino oscillation are

$$
\theta_{23}^{e, \nu} \approx 1, \quad \theta_{13}^{e, \nu} \approx \varepsilon, \quad \theta_{12}^{\nu}=45^{\circ}, \quad \theta_{12}^{e} \approx \varepsilon
$$

and

$$
\Delta m_{23}^{2} \approx \frac{1}{\varepsilon^{2}} \frac{\left(\lambda^{\prime} v\right)^{4}}{M^{2}}, \quad \Delta m_{12}^{2} \approx\left(\varepsilon+\varepsilon^{\prime}\right) \frac{\left(\lambda^{\prime} v\right)^{4}}{M^{2}} .
$$

It is remarkable that $L_{e}-L_{\mu}-L_{\tau}$ has forced a pseudo-Dirac structure in the 12 subspace as in its previous realization, again giving $\theta_{12}$ near $45^{\circ}$. The crucial difference is that the pseudo-Dirac mass splitting is now a higher power in FSB than before

$$
\frac{\Delta m_{12}^{2}}{\Delta m_{23}^{2}} \approx \varepsilon^{2}\left(\varepsilon+\varepsilon^{\prime}\right)
$$

rather than $\varepsilon+\varepsilon^{\prime}$. This allows $\varepsilon$ and $\varepsilon^{\prime}$ to be considerably larger than before, so $\sin 2 \theta_{12}<0.8$ is now possible, allowing large angle MSW solar neutrino oscillations. In this case the FSB parameters are not very small $\varepsilon, \varepsilon^{\prime} \approx 0.3 \div 0.5$, so that the mass of the right-handed neutrinos is still quite close to the gauge coupling unification scale. Notice again the cosmological irrelevance of the neutrino masses. For neutrino-less $2 \beta$ decay searches $\left(m_{L L}\right)_{11} \approx \epsilon^{3}\left(\Delta m_{23}^{2}\right)^{1 / 2} \leq 10^{-2} \mathrm{eV}^{2}$. Finally, $\varepsilon^{\prime} \approx 0.1$ and $\lambda^{\prime} \approx 1$ can make $M$ exactly coincident with the unification scale.

So far we have only produced models with large $\theta_{12}$. However $L_{e}-L_{\mu}-L_{\tau}$ realized with the seesaw mechanism may also lead to small $\theta_{12}$, using the FSB suppression factors in (6.8). Taking $\eta^{\prime} \ll \varepsilon^{\prime}$ and $\eta \approx 1$, in an appropriate 23 basis gives

$$
m_{L L}=\frac{\left(\lambda^{\prime} v\right)^{2}}{M}\left(\begin{array}{ccc}
{\eta^{\prime}}^{2} \varepsilon & \eta^{\prime} & \eta^{\prime} \\
\eta^{\prime} & \varepsilon^{\prime} & 0 \\
\eta^{\prime} & 0 & \frac{1}{\varepsilon}
\end{array}\right)
$$

so that eq.s (6.13b) and (6.13c ) remain valid but

$$
\theta_{23}^{e, \nu} \approx 1, \quad \theta_{13}^{e, \nu} \approx \eta^{\prime} \varepsilon, \quad \theta_{12}^{e} \approx \varepsilon^{\prime}
$$

and, most importantly

$$
\theta_{12}^{\nu} \approx \eta^{\prime} / \varepsilon^{\prime}
$$

which can make $\theta_{12}$ small. 


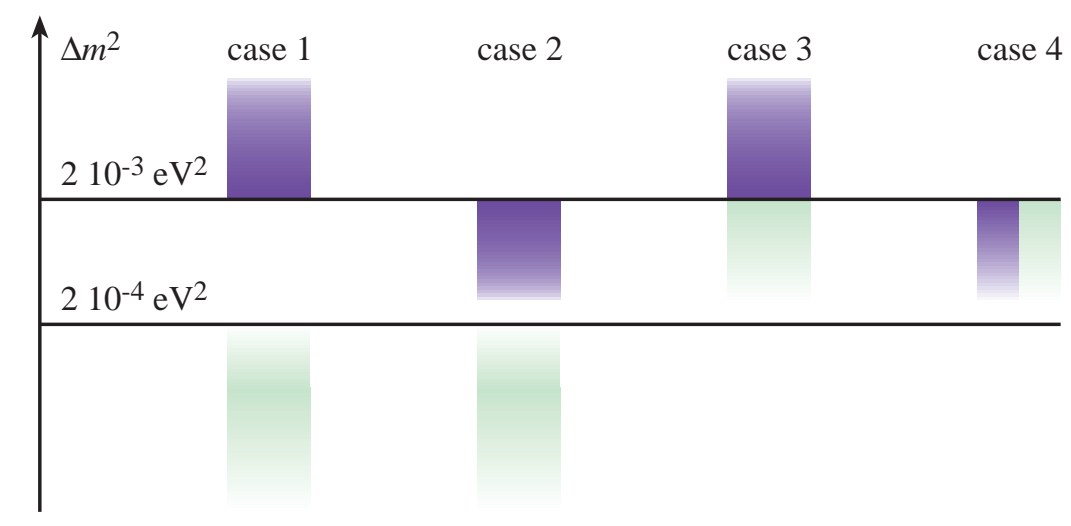

Figure 7: Different combinations of ranges for $\Delta m_{23}^{2}$ (dark gray) and $\Delta m_{12}^{2}$ (light gray) discussed in the text.

\section{Conclusions}

The solar and atmospheric neutrino anomalies, strengthened by the recent SuperKamiokande observations, can be interpreted as due to oscillations of the three known neutrinos. However there is still considerable allowed ranges of masses and mixing angles that can account for all these anomalies, especially if a cautious attitude is taken with regard to the theoretical analysis and/or the (difficult) experiments relevant to solar neutrinos. A further major element of uncertainty is related to the relatively large range of values for the mass splitting that can account for the atmospheric neutrino anomaly. We summarize our conclusions by considering a set of alternative hypotheses, related to these dominant uncertainties, with an eye to the experimental program that may lead to their resolution and eventually to the determination of the full set of neutrino oscillation parameters.

A critical value for $\Delta m_{23}^{2}$ is around $2 \cdot 10^{-3} \mathrm{eV}^{2}$ mainly because for larger values CHOOZ sets a considerable constraint on the mixing pattern, but also because $(1 \div 2) \cdot 10^{-3} \mathrm{eV}^{2}$ is frequently discussed as a typical sensitivity limit for various Long-Base-Line (LBL) neutrino experiments, like the one from KEK to SK, or the $\nu_{\tau}$ appearance experiments with a high energy beam from CERN to Gran Sasso or from Fermilab to Soudan. On the other end, a value of $\Delta m_{12}^{2}<2 \cdot 10^{-4} \mathrm{eV}^{2}$, as certainly required by a standard Solar Neutrino Analysis (SNA), would make the corresponding oscillation frequency irrelevant to the SK experiment on atmospheric neutrinos. On this basis we consider the following four possibilities, none of which, we believe, can be firmly excluded at present. They are graphically represented in fig. ㄱ.

1. $\Delta m_{23}^{2}>2 \cdot 10^{-3} \mathrm{eV}^{2}$ and $\Delta m_{12}^{2}<2 \cdot 10^{-4} \mathrm{eV}^{2}$. Here a minimal scheme to describe both solar and atmospheric neutrinos is required, as discussed in section 3. with $\Delta m_{23}^{2} \gg \Delta m_{12}^{2}$. Since $\Delta m_{12}^{2}$ is too small to affect atmospheric and/or LBL experiments, in both cases eqs. 3.3 apply. The fit relevant to SK is given in fig. 6a, with the further constraint, from CHOOZ, that $\theta_{13}$ is small, $\theta_{13} \leq 13^{\circ}$, and therefore $\theta_{23}=45^{\circ} \pm 15^{\circ}$. In turn $\theta_{12}$, together with $\Delta m_{12}^{2}$, will have to be determined by solar neutrino experiments. In this alternative, the neatest confirmation of the SK result would come from a $\nu_{\tau}$ appearance LBL experiment. At the same time, a dominant $\nu_{\mu} \rightarrow \nu_{\tau}$ oscillation should also lead to a signal in the KEK to SK $\nu_{\mu}$ disappearance experiment, with no appreciable $\nu_{e}$ appearance signal.

2. $\Delta m_{23}^{2}<2 \cdot 10^{-3} \mathrm{eV}^{2}$ and $\Delta m_{12}^{2}<2 \cdot 10^{-4} \mathrm{eV}^{2}$. The main difference with respect to the previous case is that now $\theta_{13}$ is not constrained by CHOOZ, and therefore, from fig. 6a, it can be as large as $45^{\circ}$. This implies, from eqs. 3.3 , that the results of both atmospheric and LBL experiments, with low enough $\nu_{\mu}$ energies to permit exploration of $\Delta m^{2}$ lower than $2 \cdot 10^{-3} \mathrm{eV}^{2}$, may be affected by a significant $P_{\mu e} \neq 0$. By the same token, an experiment with low energy $\bar{\nu}_{e}$ extending the sensitivity of CHOOZ (e.g. Kam-LAND) may show a large signal if $\theta_{13}$ is indeed large. In any event $P_{\mu \tau}$ will be significant. Finally, as in case 1., decoupling of solar and atmospheric neutrino oscillations implies that $\theta_{12}$ can only be determined by solar neutrino experiments, with an analysis complicated by $\theta_{13}$ being potentially unconstrained (see fig.s 3 , upper row)

3. $\Delta m_{23}^{2}>2 \cdot 10^{-3} \mathrm{eV}^{2}$ and $\Delta m_{12}^{2}>2 \cdot 10^{-4} \mathrm{eV}^{2}$. This case is possible only if SSM constraints are relaxed (fig. 3, lower row) and/or if one of the experimental techniques for solar neutrinos is problematic (fig. 5). However, as discussed in section $4 . \Delta m_{12}^{2}$ must be lower than $2 \cdot 10^{-3} \mathrm{eV}^{2}$, below the CHOOZ range. Since, on the other hand, $\Delta m_{\mathrm{atm}}^{2}=\Delta m_{23}^{2}$ is in the CHOOZ range, $\theta_{13}$ is small and eq.s 4.3 are relevant 
for atmospheric and LBL experiments. The fit of the present SK results gives $\theta_{23}=45^{\circ} \pm 25^{\circ}$ (the range at $\theta_{23} \approx 135^{\circ}$ being equivalent since $\theta_{13}$ is small). Therefore the main difference with respect to case 1 . is the possibility of a $S_{12}$ contribution in eq. (4.3). While $\nu_{\tau}$ appearance in LBL experiments must still give a positive signal, $P_{\mu e}$ could significantly deviate from zero at low enough oscillation frequencies (relevant to lower energy $\nu_{\mu}$ LBL experiments or to reactor experiments such as Kam-LAND). The finding of such an effect, together with a positive $\nu_{\tau}$ appearance signal, would prove, in the three neutrino oscillation picture, the inadequacy of the NSA as it is done now.

4. $\Delta m_{23}^{2}<2 \cdot 10^{-3} \mathrm{eV}^{2}$ and $\Delta m_{12}^{2}>2 \cdot 10^{-4} \mathrm{eV}^{2}$. This is the relatively less constrained case (and also the relatively less likely). Here both neutrino squared mass differences are outside of the CHOOZ range, so that $\theta_{13}$ is unconstrained. Appropriate values of the mixing angles can fit the SuperKamiokande up/down ratios of atmospheric neutrinos, as shown in fig. 6 b. In this case, the two comparable $\Delta m^{2}$ might lead to sizeable CP-violating effects if all the three mixing angles are large.

Measurements by SNO and Borexino will increase the number of independent observational signals of the solar fluxes, $S_{i}$, from 3 to 5 ; so that, from (2.12) with $\Phi_{\mathrm{CNO}} / \Phi_{\tau_{\mathrm{Be}}}=0.22, \Delta m_{12}^{2}, \theta_{12}, \theta_{13}, \Phi_{\tau_{\mathrm{Be}}}$ and $\Phi_{8_{\mathrm{B}}}$ can all be determined. This will provide a crucial consistency check between the experimental techniques and the solar models. If $\theta_{13}$ is found to be large, $\Delta m_{23}^{2}<2 \times 10^{-3} \mathrm{eV}^{2}$, giving a signal at Kam-LAND, but making it harder for LBL experiments.

In the minimal scheme, with a hierarchy amongst the $\Delta m^{2}$, several years of data from Super-Kamiokande will allow a fit to $\Delta m_{23}^{2}, \theta_{23}$ and $\theta_{13}$. Combining with fits to the solar flux measurements, and to LBL and Kam-LAND experiments, could allow the emergence of a consistent picture for the two oscillation frequencies and the three leptonic mixing angles.

The variety of possibilities discussed above makes it uncertain which is the relevant neutrino mass matrix and, a fortiori, which are the flavour symmetries that might be responsible for it. Nevertheless, focusing on the minimal scheme for both solar and atmospheric neutrinos, the peculiar pattern of masses and mixings renders meaningful the search for an appropriate mass matrix. As discussed in section 5 on general grounds, two forms of mass matrices emerge as being able to describe the data, eq.s (6.1) and (6.2). Since in the minimal scheme $\Delta m_{12}^{2} \ll 2 \cdot 10^{-3} \mathrm{eV}^{2}$, these forms imply that neutrino masses will not give rise to an observable neutrinoless double beta decay signal. The combination $L_{e}-L_{\mu}-L_{\tau}$ of the individual lepton numbers may play a role in yielding both these forms. A common feature of the resulting solutions is that the heaviest neutrino mass is determined by the oscillation length of the atmospheric neutrinos, $\left(\Delta m_{\mathrm{atm}}^{2}\right)^{1 / 2}$. As such, the neutrino masses are irrelevant for present cosmology. Again quite in general, an increasing separation between the two $\Delta m^{2}$ requires the angle $\theta_{13}$ to become increasingly small.

Acknowledgements We thank Zurab Berezhiani for a useful comment. This work was supported in part by the U.S. Department of Energy under Contracts DE-AC03-76SF00098, in part by the National Science Foundation under grant PHY-95-14797, in part by the TMR network under the EEC contract n. ERBFMRXCT960090.

\section{References}

[1] Y. Fukuda et al. (Super-Kamiokande collaboration), hep-ex/9805021: Y. Suzuki (Super-Kamiokande collaboration), in Neutrino 98, proceedings of the 18th international conference on neutrino physics and astrophysics, Takayama, Japan, edited by Y. Suzuki and Y. Totsuka, to appear on Nucl. Phys. B (Proc. Suppl.).

[2] J.N. Bahcall and M.H. Pinsonneault, Rev. Mod. Phys 67 (1995) 781 ("BP95"). The recent revision of these fluxes, J.N. Bahcall, S. Basu and M.H. Pinsonneault, astro-ph/9805135 ("BP98"), would not change any of our conclusions.

[3] Y. Fukuda et al. (Super-Kamiokande collaboration), hep-ex/9805006.

[4] L. Wolfenstein, Phys. Rev. D17 (1978) 2369; S.P. Mikheyev and A. Yu Smirnov, Sov. J. Nucl. Phys. 42 (1986) 913.

[5] S.P. Mikheyev and A. Yu Smirnov, Phys. Lett. B200 (1988) 560;

G. Fogli, E. Lisi and D. Montanino, Phys. Rev. D54 (1996) 2048 (hep-ph/9605273);

S. Bilenky, C. Giunti and C. Kim, Astrop. Phys. 4 (1996) 241 hep-ph/9505301);

T. Teshima, T. Sakai and O. Inagaki, hep-ph/9801276.

[6] S. Parke, Phys. Rev. Lett. 57 (1986) 1275;

T.K. Kuo and J. Pantaleone, Phys. Rev. D35 (1987) 3432. 
[7] J.N. Bahcall, "Neutrino Astrophysics", Cambridge University Press, 1989.

[8] Solar neutrino fluxes and cross sections can be obtained from the J.N. Bahcall internet page, at www.sns.ias.edu/ jnb.

[9] For a review and references see V. Castellani et al., Phys. Rep. 281 (1997) 309 astro-ph/960618d).

[10] B.T. Cleveland, Nucl. Phys. (Proc. Suppl.) B38 (1995) 47; K. Lande et al., in Neutrino '96, proceedings of the 17th international con- ference on neutrino physics and astrophysics, Helsinki, 1996, edited by K. Huitu, K. Enqvist and J. Maalampi (World Scientific, Singapore, 1997).

[11] W. Hampel et al. (Gallex collaboration), Phys. Lett. B388 (1996) 384;

Gallex Collaboration, TAUP97, Laboratori Nazionali del Gran Sasso September 1997, to appear on Nucl. Phys. B (Proc. Suppl.); V. Gavrin et al. (SAGE collaboration), Phys. Lett. B328 (1994) 234; SAGE Collaboration, Neutrino 96, Helsinki June 1996 to appear on Nucl. Phys. B (Proc. Suppl.).

[12] Y. Fukuda et al., Phys. Rev. Lett. 77 (1996) 1683.

[13] N. Hata and P. Langacker, Phys. Rev. D52 (1995) 420 (hep-ph/9409372);

N. Hata and P. Langacker, Phys. Rev. D56 (1997) 6107 (hep-ph/9705339).

[14] P.I. Krastev and S.T. Petcov, Phys. Lett. B395 (1997) 69 hep-ph/9612243).

[15] G.L. Fogli, E. Lisi and D. Montanino, hep-ph/9709473.

[16] Q.Y. Liu, M. Maris and S. Petcov, Phys. Rev. D56 (1997) 5991 (hep-ph/9702361);

M. Maris and S. Petcov, Phys. Rev. D56 (1997) 7444 (hep-ph/970539g).

[17] P. Lipari and M. Lusignuoli, hep-ph/9803440.

[18] M. Honda, T. Kajita, K. Kasahara, and S. Midorikawa, Phys. Rev. D52 (1995) 4985.

[19] M. Apollonio et. al (CHOOZ collaboration), hep-ex/9711009.

[20] V. Barger, T. J. Weiler, K. Whisnant, hep-ph/9807319:

G.L. Fogli, E. Lisi, A. Marrone, G. Scioscia, hep-ph/9808203.

[21] C. Athanassopoulos et al. (LSND collaboration) Phys. Rev. Lett. 75 (1995) 365; ibid. 77 (1996) 3082, nucl$e x / 9706000$

[22] P. Binetruy et al., Nucl. Phys. B496 (1997) 3 (hep-ph/9610481); M. Fukugita, M. Tanimoto and T. Yanagida, Phys. Rev. D57 (1998) 4429 hep-ph/9709388); Zhi-zhong Xing, hep-ph/9804433.

[23] M. Drees et al., Phys. Rev. D57 (1998) 5335 hep-ph/9712392); M. Bando, T. Kugo, K. Yoshioka, Phys. Rev. Lett. 80 (1998) 3004 (hep-ph/9710417); V. Barger, S. Pakvasa, T. J. Weiler, K. Whisnant, hep-ph/9806387; S. King, hep-ph/9806440.

[24] M. Gell-Mann, P. Ramond, and R. Slansky, in Supergravity (ed. P. van Nieuwenhuizen and D. Z. Freedman, NorthHolland, Amsterdam, 1979), p. 315;

T. Yanagida, in proceedings of the workshop on the unified theory and the baryon number in the universe (ed. O. Sawada and A. Sugamoto, KEK Report No. 79-18, Tsukuba, Japan, 1979). 